\title{
Tradeoffs in optimal control capture patterns of human sensorimotor control and adaptation
}

Tyler Cluff ${ }^{1,4,5}$, Frederic Crevecoeur ${ }^{1,6,7}$, Stephen H. Scott ${ }^{1,2,3}$

${ }^{1}$ Centre for Neuroscience Studies, ${ }^{2}$ Dept. of Biomedical and Molecular Sciences, and ${ }^{3}$ Dept. of Medicine,

\section{Queen's University, Kingston, ON, Canada}

${ }^{4}$ Faculty of Kinesiology, ${ }^{5}$ Hotchkiss Brain Institute, University of Calgary, Calgary, AB, Canada

'Institute of Information and Communication Technologies, Electronics and Applied Mathematics, ${ }^{7}$ Institute of Neuroscience, University of Louvain, Belgium (UCLouvain)

Pages: 52 (including references, figures, and methods)

Figures: 7

Tables: 1

Word Count:

Abstract: 200

Main Text: 6285

Figure Captions: 844

Keywords: reaching, motor adaptation, optimal control theory, force-field adaptation, mechanical perturbations, muscle stretch responses, feedback control.

\section{Please address correspondence to:}

Stephen H. Scott

Laboratory of Integrative Motor Behaviour

Centre for Neuroscience Studies

18 Stuart St., Queen's University

Kingston, ON, K7L 3N6, Canada

Phone: +1-613-533-2855

Fax: +1-613-533-6840

Email: steve.scott@queensu.ca 


\section{Abstract}

48

49 Modern control theory highlights strategies that consider a range of factors, such as errors caused by environmental disturbances or inaccurate estimates of body or

51 environmental dynamics. Here we reveal similar diversity in how humans naturally

52 adapt and control their arm movements. We divided participants into groups based

53 on how well they adapted to interaction loads during a single session of reaching

54 movements. This classification revealed differences in how participants controlled

55 their movements and responded to mechanical perturbations. Interestingly,

56 variation in behaviour across good and partial adapters resembled simulations from

57 stochastic and robust optimal feedback control, respectively, where the latter

58 minimizes the effect of disturbances, including those introduced by inaccurate 59 internal models of movement dynamics. In a second experiment, we varied the 60 interaction loads over short time periods making it difficult to adapt. Under these 61 conditions, participants who otherwise adapted well altered their behaviour and 62 more closely resembled those using a robust control strategy. Taken together, the 63 results suggest the diversity of how humans control and adapt their arm movements 64 may reflect the accuracy of (or confidence in) their internal models. Our findings may open novel perspectives for interpreting motor behaviour in uncertain environments, or when neurologic dysfunction compromises motor adaptation. 


\section{Introduction}

72 Humans can perform a range of motor skills, from playing piano to throwing

73 a baseball, but vary in how well they learn to perform these skills. Although it is

74 widely accepted by coaches and music instructors that motor learning patterns vary

75 between individuals ${ }^{1-5}$, studies typically focus on how factors, such as the structure

76 or timing of practice, influence average patterns of adaptation across groups of

77 participants ${ }^{6-8}$. This approach has had implications for how motor skills are taught ${ }^{1}$

78 and rehabilitated after neurologic injuries, disorders and diseases ${ }^{9}$.

79 Studies are only beginning to reveal the diversity of sensorimotor adaptation.

80 It is becoming clear that healthy individuals differ in the rate they adapt their

81 reaching movements when exposed to novel mechanical loads or visual

82 disturbances within a single experimental session ${ }^{10-15}$. Interestingly, individuals

83 who make the largest errors when first exposed to novel force disturbances tend to

84 learn faster ${ }^{12}$. The authors argued that larger movement errors may accelerate

85 adaptation by providing a better teaching signal. However, it is unclear why initial

86 movement errors differ between individuals when exposed to the same visual or

87 mechanical disturbances.

88 Healthy individuals also differ in the amount they adapt their reaching

89 movements when they encounter novel mechanical loads. Although participants

90 attain approximately $80 \%$ adaptation, on average, within minutes to hours of being

91 exposed to novel movement dynamics, some participants display near-complete

92 adaptation and others only partially adapt their movements with the same amount

93 of practice ${ }^{16-19}$. Simulation studies suggest the nervous system is less sensitive to 
94 noise that corrupts motor commands or sensory receptors than it is to errors in its

95 internal models of movement dynamics ${ }^{20}$. Indeed, even small internal model errors

96 can produce unstable movements when moving in the presence of novel dynamics

97 or external perturbations ${ }^{21}$. An emerging idea is that the nervous system may

98 increase sensitivity to visual or somatosensory feedback to deal with incomplete

99 learning 22,23 , but it is unclear why and under what circumstances this strategy is 100 beneficial for performing reaching movements.

101 Towards filling this gap, we demonstrate that principles of optimal feedback

102 control capture tradeoffs in how people reach and respond to novel movement

103 dynamics. Across two experiments, we demonstrate that patterns of human

104 sensorimotor control and adaptation, measured in a single session of reaching

105 movements, broadly resemble stochastic optimal feedback control and robust

106 optimal feedback control. These two variants of optimal control differ in terms of

107 how the controllers handle errors and uncertainty in their internal models of the

108 body and environment. Taken together, the results and simulations illustrate how

109 the accuracy of (or confidence in) internal models of the body and environment may

110 influence the control strategies individuals select to interact with their environment.

114

115

116 


\section{Results}

118 Experiment 1. Learning to move in the presence of novel interaction loads

119 We examined how healthy participants $(N=40$; right handed) adapted their

120 reaching movements when exposed to novel interaction loads. Participants first

121 completed unloaded movements (Baseline) to three targets presented in random

122 order $(\mathrm{T} 1-\mathrm{T} 3$, radius $=1 \mathrm{~cm}$; Fig.1A). We positioned the targets so that the arm

123 displacements required to reach them were identical across participants. Target 1

124 required shoulder motion (T1), Target 2 required combined shoulder and elbow

125 motion (T2), and Target 3 required elbow motion (T3). We then introduced novel

126 shoulder-joint loads (Fig.1 A-B; Adaptation) that were proportional to the angular

127 velocity of the elbow joint (i.e., interaction loads) ${ }^{24}$. The interaction loads

128 systematically disturbed the participant's arm while they reached the training

129 targets (T2-T3). Participants were instructed to reach the targets within 450-650

130 ms. Movement durations were determined as the time between when the

131 participant's hand left the start position (radius $=0.625 \mathrm{~cm}$ ) to the time it entered

132 the end target. The end target turned green to indicate successful timing, and blue

133 (too slow) or red (too fast) if participants did not meet the timing demands.

134 Participants also reached to a separate test target (T1) that required only

135 shoulder flexion motion. We probed feedback responses by randomly disturbing

136 the arm with step-torque perturbations that were applied the instant the participant's

137 hand left the start position (20\% of trials at T1). The step-torque perturbations

138 produced elbow joint extension and required a rapid correction to reach the target

139 within the time constraints (-2Nm at shoulder and elbow; 10 ms sigmoidal ramp-to- 
140 constant profile $)^{25}$. The same time constraints and performance feedback were

141 used for unperturbed and perturbed reaching trials.

142 Elbow motion while reaching to T1 was limited $\left(<2^{\circ}\right)$ and produced small,

143 variable shoulder-joint loads during the adaptation phase (integrated shoulder load

144 at T1 $<3 \%$ of loads at T2-T3). In principle, changes in muscle activity were not

145 required to reach T1 when moving in the presence of the interaction loads. This

146 design allowed us to probe feedback responses while minimizing the automatic

147 gain scaling of muscle stretch responses ${ }^{26-29}$ caused by increases in muscle activity

148 when countering the interaction loads to reach the training targets (T2-T3). In short,

149 the design allowed us to test whether the amount participants compensated for the

150 interaction loads while reaching the training targets (T2-T3) influenced how they

151 responded to step-torque perturbations while reaching the test target (T1).

152 We first separated participants into two groups based on how well they 153 compensated for the novel interaction loads. This metric captures systematic

154 deviations in the hand paths of individual participants that reflect the accuracy of

155 their internal models of arm and environmental dynamics $8,16,23,30-34$. We classified

156 participants as 'good adapters' if their peak hand-path deviations returned to near

157 baseline levels by the end of training (Fig.1 C-D, single-sided $t$-test performed on

158 individual participant data, $p>0.05)$. Participants were classified as 'partial

159 adapters' if their hand path deviations remained larger than baseline levels $(p<$

1600.05 for individual participants). As a group, good adapters displayed near complete

161 adaptation $(\mathrm{n}=27 ; 94.7 \pm 3.9 \%$ ), and partial adapters displayed incomplete

162 adaptation $(\mathrm{n}=13 ; 72.2 \pm 4.9 \%$, Supplementary Fig. 1$)$. 
163 Consistent with more accurate internal models of the novel interaction loads,

164 good adapters displayed a larger increase in the agonist burst of their pectoralis

165 major muscle (PM, shoulder flexor) while reaching target 3 (T3; Supplementary Fig.

166 2). The relationship between EMG bursts at the shoulder and elbow is well

167 characterized for single-joint elbow movements ${ }^{35-37}$. Under natural conditions,

168 elbow flexion motion produces an extension torque at the shoulder. When planning

169 single-joint elbow movements, the nervous system counters extensor interaction

170 torques by producing anticipatory activity of the shoulder flexor muscles. The

171 interaction dynamics in our experiment accentuated the shoulder extensor torques

172 produced during elbow flexion. Given that brachioradialis EMG (elbow flexor) was

173 relatively constant during adaptation, the larger average increase in pectoralis

174 major EMG displayed by good adapters suggests they exploited an internal model

175 of the interaction dynamics.

176 Interestingly, we found that good adapters, who displayed near-complete

177 adaptation to novel shoulder loads, also displayed larger peak hand-path deviations

178 than partial adapters when the novel loads were first introduced (Fig. 1E, T2: $t(38)$

$179=2.18, p=0.018$, T3: $t(38)=2.45, p=0.0096)$. Similar results were observed when

180 broadening the analysis to the first 5 loaded movements to each training target (T2:

$181 t(38)=1.88, p=0.034 ;$ T3: $t(38)=1.81, p=0.040)$. Despite some differences in

182 peak shoulder loads, the overall mechanical demands of the task were qualitatively

183 similar between good and partial adapters (Supplementary Fig. 3). Inertial

184 properties of the arm were also similar across groups of good and partial adapters. 
exposed to novel interaction loads and the extent to which adaptive processes

187 compensate for these loads. Does this link reflect differences in the way participants

188 control and adapt their reaching movements?

189 Optimal control theory may provide a framework to understand the link

190 between initial and final performance, as there are many factors for the nervous

191 system to consider when moving in the presence of novel dynamics. An influential

192 theory for interpreting the function of the sensorimotor system is stochastic optimal

193 feedback control (known as Linear-Quadratic-Gaussian, or extended LQG-

194 control $\left.^{38}\right)$. Stochastic optimal control uses a control policy to generate motor

195 commands that represent the best way to perform an action (e.g., most accurate

196 with minimal effort), assuming the controller's internal model of arm and

197 environmental dynamics is accurate (i.e., unbiased) and noise in the motor system

198 follows a known distribution (Gaussian noise in the context of LQG). Even small

199 biases in the internal model can cause movement errors when a stochastic optimal

200 controller encounters loads that alter the dynamics of the arm or environment ${ }^{39}$.

201 Another class of optimal control models explicitly accounts for potential

202 errors in the internal model when finding a solution for a motor task: robust optimal

203 feedback control, which we formulate here in the framework of $H_{\infty}$-control ${ }^{40,41}$. The

204 principle in robust control is to set the feedback gains so that the control policy is

205 as insensitive to disturbances as possible, including disturbances caused by biases

206 or uncertainty in the internal model of body and environmental dynamics (i.e., the

207 biological plant). Though insensitive to disturbances, robust control increases the 
208 cost of movement because the controller responds to all movement disturbances,

209 regardless of how small or transient ${ }^{41}$. A stochastic optimal feedback controller

210 ignores these deviations following the assumption it makes about the random (i.e.,

211 stochastic) nature of the disturbances.

212 In short, the control signals produced by stochastic optimal feedback control

213 are less affected by noisy motor commands because the controller assumes noise

214 disturbances are zero on average. The stochastic optimal feedback controller is

215 also coupled with a minimum variance estimator (Kalman filter) that filters out noise

216 in motor commands and sensory feedback when estimating the state of the arm.

217 This allows the controller to perform efficient movements at the cost of making

218 larger errors when the dynamics of the body or environment change unexpectedly.

219 Robust control instead views any unmodeled disturbance as a perturbation, which

220 results in control that is less efficient but also less sensitive to changes in the

221 dynamics of the body or environment. In essence, stochastic and robust optimal

222 feedback control lie at the extremes of a continuum of control strategies that differ

223 in terms of how efficient versus sensitive the controller is to errors in its internal

224 models of body and environmental dynamics.

225 Good and partial adapters were exposed to the same novel interaction loads

226 but displayed differences in their average adaptation patterns. Within the context of

227 stochastic and robust optimal feedback control, the observation that good adapters

228 made larger initial errors but on average attained better final performance suggests

229 they relied on their internal models of movement dynamics. This strategy would

230 only be viable if they were capable of updating their internal model when confronted 
231 with interaction loads that altered the dynamics of the arm and environment. As a

232 group, partial adapters appeared to compensate for the incomplete adaptation of

233 their internal models by using a control strategy that was more robust to (potential)

234 movement disturbances. By definition, robust control is less sensitive to internal

235 model errors. This strategy would allow partial adapters to make smaller errors

236 when the interaction loads were first introduced, as well as mitigate the impact of

237 internal model errors throughout the (incomplete) adaptation process. Taken

238 together, our data highlight a potential link between the amount participants adapt

239 and the robustness of the strategy they select to control their reaching movements.

240 We formalized this link using a simplified model of the arm reaching to the

241 same targets with the same time and accuracy demands as our human participants

242 (Online Methods). The arm was controlled by stochastic and robust optimal

243 feedback controllers paired with state estimators that helped circumvent the effect

244 of noisy motor commands and noisy, time-delayed sensory feedback. Noise and

245 cost parameters were taken from the literature or selected to generate motion

246 patterns that qualitatively resembled human behaviour (see Table 1).

248 Unperturbed Baseline Movements

249 Owing to its increased control gains, the robust controller produced baseline

250 movements with faster and earlier peak hand velocities than the stochastic optimal

251 controller (Fig. 2A). The robust optimal controller also generated more variable

252 hand motion profiles than the stochastic optimal controller (Fig. 2A). Recall the two

253 control models use the same cost function, time to complete the movement, and 
254 state space representation of the arm but solve the control problem in different

255 ways. Increased variability emerges from the robust controller's sensitivity to motor

256 noise (process noise). Unlike stochastic optimal control, the robust controller does

257 not assume disturbances are zero on average. Thus, the robust controller does not

258 filter out disturbances arising from noise and treats any disturbance as a 259 perturbation.

260 Interestingly, we found the movements of robust and stochastic optimal

261 controllers were paralleled by the behaviour of partial and good adapters. Partial

262 adapters displayed faster peak hand velocities (Fig.2B, T1-T3: all $t(38)>3.05$; all $p$

$263<0.01$ ) and more variable movements than good adapters in the baseline phase of

264 the experiment (T1-T3: all $t(38)>1.98$, all $p<0.05)$.

265

266

\section{Responses to Step-Torque Perturbations During Baseline Movements}

Stochastic and robust optimal feedback controllers make fundamentally different assumptions about disturbances that arise during movement. Thus, we applied step-torque perturbations to view how the controllers responded to unexpected disturbances (Fig. 3A). The robust controller generated rapid and large

271 corrective responses (Fig. 3C) to counter the perturbation and reach the target (Fig.

$2723 B$ ). When disturbed by the same step torque, the stochastic optimal controller 273 generated larger control responses than the robust controller but later in movement 274 (Fig. 3C). As a result, it displayed larger hand-path deviations and subsequent goal275 directed corrections (Fig. 3B).

276 The behaviour of partial and good adapters paralleled how the robust and 277 stochastic optimal controllers responded to step-torque disturbances, respectively. 
278 When their arm was disturbed by the step torque, partial adapters displayed smaller

279 average peak hand deviations than good adapters (Fig. 3D, $t(38)=2.45, p<0.01$ ),

280 and larger pectoralis major (PM) activity in the long-latency and voluntary time 281 windows (Fig. 3E, LL $=50-105$ ms post-perturbation, $t(38)=2.05, p<0.05$; VOL $=$ $282105-180 \mathrm{~ms}, t(38)=2.22, p<0.01)$. Note that differences in how the robust and 283 stochastic optimal controllers responded to the step-torque perturbation emerged 284 earlier (50-100 ms) than in the muscle responses of good and partial adapters ( $75-$ $285150 \mathrm{~ms}$ ). Although our simplified model of muscle contraction (low pass filtered 286 version of the control command) produces faster behavioural corrections, the EMG 287 and behavioural results are consistent with the rise time of neural signals and 288 evidence that it takes $>100$ ms for humans to reach peak muscle activity when 289 instructed to move as quickly as possible ${ }^{42}$. Muscle stretch responses were broadly 290 consistent with model control signals at longer time scales $(300+\mathrm{ms}$; 291 Supplementary Fig. 4).

292

\section{Adaptation to Novel Interaction Loads}

294 Unperturbed Voluntary Movements

Next, we return to the issue of adaptation to novel interaction loads, as 296 displayed in Figure 1C-D for human participants. Both controllers deviated from 297 their baseline movements when moving with inaccurate knowledge of the novel 298 interaction loads. However, the robust controller displayed smaller deviations than 299 the stochastic optimal controller, just as partial adapters displayed smaller average 300 hand path deviations than good adapters on the first adaptation trial (Fig.1C-D). We 301 manipulated the accuracy of each controller's internal model to examine how 
302 sensitive it was to imperfect knowledge of the interaction dynamics. The robust

303 controller always displayed smaller peak hand path deviations than the stochastic

304 optimal controller when moving with an inaccurate internal model of arm and

305 environmental dynamics (Supplementary Fig. 5).

306

307 Feedback Corrections in the Presence of Novel Interaction Dynamics

We next explored how the controllers responded to step-torque perturbations

when first exposed to novel interaction loads. When applied during baseline

movements to T1, the step-torque extended the elbow while minimizing shoulder

311 motion $^{43}$. Combined elbow and shoulder flexor torques were required to counter the

312 perturbation and reach the target. Recall the interaction loads produce shoulder

313 loads proportional to the elbow's angular velocity (elbow flexion causes shoulder

314 extensor load). Elbow motion while reaching to T1 was limited $\left(<2^{\circ}\right)$ and produced

315 small loads during reaching ( $<3 \%$ of load at T2-T3). When the same step torque

316 was applied during adaptation, larger shoulder flexor torques were required to

317 compensate for the extensor loads produced when countering the perturbation and

318 flexing the elbow to reach the target. This approach allowed us to probe each

319 controller's sensitivity to disturbances and reliance on its internal model.

320 Introducing the novel load produced systematic errors in the internal models

321 used by robust and stochastic optimal controllers. Internal model errors caused

322 unexpected shoulder flexion motion when the step torque extended the elbow while

323 moving in the presence of novel interaction loads. This caused both controllers to

324 reduce their shoulder flexor response (Supplementary Fig. 6). Partial and good 
325 adapters also reduced their shoulder flexor responses when disturbed by the step-

326 torque perturbation during initial adaptation trials (Supplementary Fig. 6).

327 We then compared model performance with human behaviour at the end of

328 the adaptation block. For comparison, we constrained knowledge of the interaction

329 load in our model simulations to the amount of adaptation displayed by our sample

330 of good and partial adapters (Supplementary Fig. 7). Recall that good adapters,

331 who better updated their internal models to compensate for interaction loads,

332 displayed a larger average increase in shoulder flexor activity when producing pure

333 elbow movements (T3). The stochastic optimal feedback controller also leveraged

334 its adapted internal model. Before the onset of movement, it compensated for the

335 expected interaction loads by producing a larger increase in shoulder flexor torque

336 (relative and absolute) than the robust controller. This allowed the stochastic

337 optimal controller to minimize movement disturbances while reaching target 3 (T3)

338 in the presence of novel interaction loads (Supplementary Fig. 8). In short, the

339 behaviour and control commands generated by robust and stochastic optimal

340 controllers were broadly consistent with the behaviour of partial and good adapters.

341 Differences in the adapted internal models of the stochastic and robust

342 optimal feedback controllers influenced how they responded to step-torque

343 disturbances. Due to its sensitivity to motion disturbances and partially adapted

344 internal model, the robust controller decreased its shoulder flexor response when

345 the step-torque extended the elbow and produced unexpected shoulder flexion

346 motion. In contrast, the stochastic optimal controller's internal model was more

347 accurate and it generated a larger shoulder flexor response to account for extensor 
348 torques produced when countering the step-torque and flexing the elbow to reach

349 the target. As a result, it was less disturbed by the perturbation than the robust

350 controller (Fig.4A-B). In short, the robust controller produced control signals that

351 were more sensitive to individual patterns of joint motion when moving in the

352 presence of novel interaction dynamics. Stochastic optimal control generated

353 responses that were sensitive to the estimated inter-joint dynamics.

354 The model responses were paralleled by how good and partial adapters

355 responded to step-torque perturbations at the end of the adaptation phase. On

356 average, good adapters increased their PM responses (Fig. 4D, LL \& VOL time

357 windows, $t(26)>2.73, p<0.01$ ) to counter shoulder extensor loads produced when

358 correcting elbow motion. In contrast, partial adapters still reduced their PM

359 responses at the end of the adaptation phase ( $L L \&$ VOL, $t(12)>2.26, p<0.01$;

360 elbow responses in Supplementary Fig. 9). Good adapters displayed adapted hand

361 paths that were disturbed less by the step-torque perturbations than those

362 displayed by partial adapters (Fig.4C, $t(38)=2.86, p<0.01)$.

363

364 Experiment 2. Dealing with Errors Caused by Abrupt Changes in Dynamics

365 The results of Experiment 1 show that, on average, participants who

366 compensated less for novel interaction loads seemed to rely on feedback about

367 individual patterns of joint motion rather than generating responses suitable for the

368 dynamics when reaching and responding to step-torque disturbances. Previous

369 studies have reported a decrease in learning rates and increased reliance on

370 sensory feedback when moving in unpredictable dynamical environments ${ }^{22,44}$. 
371 Motivated by these results, we performed a second experiment to test whether

372 participants would make their strategy more robust when exposed to interaction

373 dynamics that changed frequently. The rapid changes in dynamics would induce

374 frequent internal model errors and make it challenging to produce accurate

375 movements when relying on a stochastic optimal feedback control strategy. This

376 control strategy is sensitive to changes in dynamics because it does not account

377 for errors or uncertainty in internal models. Thus, if good adapters use such a

378 strategy, we expected that frequent and abrupt changes in dynamics would have a

379 larger impact on their behaviour compared to that of partial adapters.

381 Adaptation to constant interaction dynamics

382

Participants were initially exposed to the same interaction load as

383 Experiment 1 (Fig. 5A). Our first step was to determine whether they behaved and 384 adapted in similar ways as participants in Experiment 1. Figure 5B shows 385 representative hand paths from participants who displayed partial and near386 complete compensation for the interaction loads. Taking the same approach as 387 Experiment 1, we separated participants into groups based on how well they 388 compensated for the interaction loads. This analysis reproduced the results of 389 Experiment 1. Good adapters (Fig. 5C, $\mathrm{n}=18 ; 92.3 \pm 6.9 \%$ ) better compensated 390 for the interaction loads than partial adapters $(n=12 ; 76.1 \pm 5.5 \%$, Supplementary 391 Fig. 10). Consistent with Experiment 1, good adapters also made larger errors when 392 the interaction loads were first introduced (Fig. 5D, First trial at T2-T3: all $t(28)>$ 393 1.88, $p<0.05$, First 5 trials at T2-T3: all $t(28)>2.71, p<0.05$ ). 
We also examined the baseline hand velocity profiles of good and partial adapters. Recall that in Experiment 1, partial adapters displayed faster, earlier

396 peaks in their hand velocity profiles than good adapters. Consistent with

397 Experiment 1, partial adapters again displayed faster and earlier peaks in their hand 398 velocity profiles than good adapters (Fig. 5E, T3: $t(28)=4.77 ; p<0.001$ ).

We probed the feedback responses of good and partial adapters by measuring the amplitude of their muscle responses when disturbed by the same 401 step-torque perturbations used in Experiment 1 (20\% of trials at T1). Partial 402 adapters again displayed larger baseline PM responses than good adapters when 403 their arm was disturbed by a step-torque perturbation ( $L L \&$ VOL, all $\mathrm{t}(28)>2.20, p$ $404<0.05)$. As in Experiment 1, good adapters leveraged their internal model when 405 responding to the step torque during the adaptation phase. They increased their 406 PM responses (Fig. 5F-G, LL \& VOL, all $t(17)>2.89, p<0.01$ ) in anticipation of 407 shoulder extensor loads produced when countering the step torque (i.e., flexing the 408 elbow) to reach the target. Partial adapters reduced their PM responses during 409 adaptation (LL \& VOL, all $t(11)<2.36, p<0.05)$

410 The classification criterion separated participants into two groups by design.

411 However, this was only for analysis purpose and it is clear that the range of 412 behavioural traits is by no means dichotomous. To show this, we investigated the 413 relationship between the amount participants in Experiments $1(n=40)$ and $2(n=$ 41430 ) adapted their hand paths (\%adaptation) and modulated their PM stretch 415 responses between the baseline and late phases of adaptation ( $\triangle E M G)$. We found 416 a significant correlation between the amount participants adapted their movements 
417 and changes in their PM responses (Fig. 6; $\mathrm{R}^{2}=0.44, p<0.001$, intercept $=-2.35$,

418 slope $\left.=0.031, F_{1,68}=53, p<0.001\right)$.

Adapting to abrupt changes in interaction dynamics

After participants completed the adaptation phase, we varied the load

422 coefficient after each block of 11 trials (Fig. 7A, 1.05-1.95 Nm.s/rad, mean load

423 coefficient $=1.5 \mathrm{Nm} . \mathrm{s} / \mathrm{rad}$ ). We probed feedback responses with the same step-

424 torque perturbations (-2 Nm, $10 \mathrm{~ms}$ sigmoidal ramp; $20 \%$ of trials at T1).

425 Consistent with our hypothesis, good adapters made larger errors when first

426 exposed to new interaction dynamics (Fig. 7B, T2-T3, $t(28)>2.17, p<0.05$ ).

427 However, their hand paths became similar to those displayed by partial adapters

428 after prolonged exposure to changes in interaction dynamics (Fig. 7B, T2-T3, $t(28)$

$429<0.29, p>0.49)$. Good adapters increased and shifted their peak hand velocity to 430 earlier in movement. Thus, their hand velocity profile became more similar to the

431 profile displayed by partial adapters (Fig. $7 \mathrm{C}, t(28)=0.33, p=0.74$ ) and predicted

432 by robust optimal feedback control.

433 When their arm was disturbed by the step-torque perturbation while reaching 434 to target 1 (T1), partial adapters showed little change in their PM responses 435 compared to when exposed to constant interaction dynamics (Fig. 7D, LL \& VOL, 436 all $t(11)<1.81, p>0.18)$. In contrast, good adapters reduced their PM responses 437 (Fig. 7E, LL \& VOL, all $t(17)>3.84, p<0.01)$. Good adapters also increased the 438 amplitude of their brachioradialis responses (Supplementary Figure 11, LL: $t(17)=$ 439 2.36, $p=0.029$, VOL: $t(17)=1.86, p=0.08)$, suggesting their control strategy 
440 became less dependent on multi-joint motion. Thus, participants seemed to use a

441 robust-like strategy when dealing with an environment that changed frequently and

442 caused internal model errors.

443

444 Discussion

445 In agreement with a growing body of literature for review see $^{45}$, we found large 446 variation in how healthy adults adapted their arm movements to counter novel 447 interaction loads. In order to understand how this variation impacted the control of 448 reaching movements, we classified participants as good or partial adapters based

449 on how well they compensated for novel interaction loads. This approach captured 450 several features of behaviour. As a group, partial adapters made smaller errors than 451 good adapters when exposed to novel interaction loads. During baseline 452 movements, partial adapters displayed faster peak hand velocities, as well as larger 453 muscle responses when disturbed by step-torque perturbations. These variable 454 patterns of sensorimotor control and adaptation were observed despite identical 455 task instructions, performance feedback, similar mechanical properties of the arm 456 and overall demands of the task.

457 The behaviour displayed by good and partial adapters was broadly consistent 458 with stochastic and robust optimal feedback control, respectively. The control 459 models had identical physical properties, noise parameters, feedback delays and 460 cost functions, but make different assumptions about disturbances that arise during 461 movement (inclusion or not of potential internal model bias or uncertainty). 462 Collectively, our models and data highlight a tradeoff between the efficiency of the 
463 control strategy and how sensitive it is to disturbances, including those introduced

464 by imperfect or uncertain internal models of movement dynamics.

465 We believe the diversity of control and adaptation patterns reflects the extent

466 to which participants relied on model-based control of their reaching movements.

467 Previous work has shown that relying on internal models can cause larger errors 468 when the task changes unexpectedly ${ }^{10}$. Consistent with this argument, introducing 469 novel interaction dynamics caused good adapters to make larger movement 470 deviations than partial adapters, who seemed to mitigate internal model errors (or 471 lack of confidence in their internal models) by relying more on feedback about the 472 motion of their shoulder and elbow joints. Strategies play a role in how people learn 473 and perform cognitive skills ${ }^{46-49}$ and make decisions ${ }^{50,51}$. We highlight that 474 strategies may also play a role in how people adapt and control their arm 475 movements when briefly exposed to novel movement dynamics.

476 Our results reveal a link between the adaptation and control of movement 477 dynamics. Variable patterns of adaptation have also been reported when healthy 478 adults receive offline error feedback while adapting their reaching movements to a

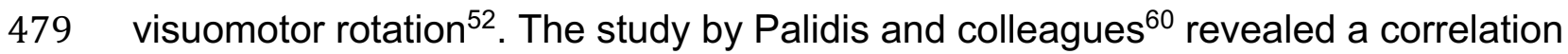
480 between learning rates and the amplitude of error-related potentials recorded over 481 parietal cortex. The authors argued that this correlation may stem from sensory 482 prediction errors, such that individuals who adapted more rapidly to the visuomotor 483 rotation relied on internal models to estimate the outcomes of their actions. 484 Moreover, a recent study demonstrated that error-related muscle activity correlates 485 with how much participants update their next movement when exposed to a force 
486 field ${ }^{12}$. Collectively, these findings suggest individuals who rely more on model-

487 based control use error-related feedback to better adapt their reaching movements.

488 Our second experiment examined whether participants would alter their control

489 strategies when exposed to less predictable dynamics. We found that good

490 adapters made larger errors than partial adapters when first exposed to

491 unpredictable dynamics, but eventually attained similar performance. Moreover,

492 good adapters increased their movement velocity and displayed patterns of

493 behaviour that more closely resembled robust control. Similar changes in

494 movement velocity have been reported when environmental forces vary

495 unpredictably between trials ${ }^{53}$. When disturbed by a step torque, good adapters

496 displayed increased control gains and shoulder responses that depended less on

497 the imposed relationship between elbow and shoulder motion and more on

498 individual patterns of joint motion.

499 Robust control is a costly but cautious strategy. The apparent change in

500 strategy highlighted in Experiment 2 suggests good adapters were less confident

501 in their estimate of the changing interaction dynamics. However, their shoulder

502 responses were larger (and elbow responses smaller) than those displayed by

503 partial adapters. This suggests participants made their strategy more robust but still

504 leveraged knowledge of the interaction dynamics - though to a lesser extent. Taken

505 together, our findings support the idea that people rely more on sensory feedback

506 when interacting with less predictable movement dynamics ${ }^{22,54,55}$.

507 Our experiments examined how participants controlled and adapted their 508 reaching movements while interacting with a robot in a single session of reaching 
509 movements. Thus, we may only provide a static snapshot of tradeoffs between

510 learning and control. This approach is common in studies that examine how

511 humans adapt their motor actions ${ }^{56-58}$, respond to mechanical disturbances ${ }^{59-61}$,

512 and shape beliefs about whether errors arise from changes in properties of the body

513 or environment ${ }^{62}$ within a brief period of practice. One exception is the work by

514 Nezafat and colleagues ${ }^{63}$ who measured force-field adaptation over 4 weeks.

515 Interestingly, the activity of the deep cerebellar nuclei changed throughout

516 adaptation despite behaviour reaching a steady state within the first practice

517 session. This raises the question of whether participants can improve their internal

518 models and alter their control strategies (particularly partial adapters), or instead

519 display stable patterns of learning and control with long term adaptation. It is also

520 unclear whether similar tradeoffs apply in tasks that depend less on dynamics (e.g.,

521 playing piano) or are performed outside of the laboratory.

522 The ability to adapt internal models may not always transfer to other aspects of

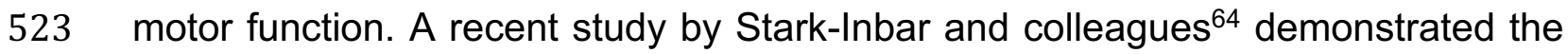

524 rate individuals adapt their movements when exposed to a visuomotor rotation does

525 not correlate with how fast they learn motor sequences. Evidence suggests these

526 tasks rely on different neural substrates and learning mechanisms ${ }^{65}$. Although it is

527 well-established that visuomotor and force-field adaptation rely on error-based

528 mechanisms, they impose different control problems for the nervous system. In

529 principle, robust strategies can be developed for a broad class of disturbances.

530 Dependent on the nature of internal models of cursor motion, participants may

531 display similar variation in the control strategies they select when adapting to 
532 visuomotor rotations. Indeed, recent evidence suggests participants may

533 upregulate their feedback responses when exposed to a visuomotor rotation ${ }^{66}$.

534 Interestingly, slower learners displayed larger spinal reflexes when their arm was

535 disturbed by a mechanical perturbation. The extent to which increased gains arise

536 from uncertain or inaccurate internal models of cursor motion and to what extent

537 control strategies can be altered by instruction are open questions for future work.

538 We argue that the behaviour of good and partial adapters align with features of

539 feedback control strategies that make different assumptions about movement

540 disturbances. Previous studies have argued the nervous system regulates the

541 limb's intrinsic resistance to disturbances (i.e., impedance) by modulating muscle

542 co-activation in a way that depends on the direction and stability of novel force

543 environments ${ }^{34,67-71}$. Here we found that long-latency responses varied with the

544 accuracy of an individual's internal model of the interaction dynamics, which caused

545 partial adapters to decrease their pectoralis major stretch responses due to

546 unexpected shoulder motion. Importantly, this result is referenced to each

547 participant's baseline stretch responses, suggesting partial adapters used a

548 strategy that was more robust (on average) and driven by increased feedback gains

549 rather than limb impedance. Standard approaches also calculate the arm's

550 impedance by averaging motion over time periods as long as $300 \mathrm{~ms}$ after the onset

551 of a perturbation ${ }^{71}$. Thus, it is difficult to attribute changes in force responses that

552 involve neural feedback gains ${ }^{72-76}$ solely to changes in the arm's intrinsic stiffness

553 or damping. The current results revealed that partial adapters displayed higher

554 levels of muscle activity. However, past work has shown that moderate to high 
555 levels of co-contraction cause only small changes in the arm's impedance ${ }^{39}$. When

556 disturbed by a perturbation, the authors reported differences in joint motion that

557 emerged $>80 \mathrm{~ms}$ after perturbation onset and correlated with the amplitude of

558 neural feedback responses involving the spinal cord and brain. In short, our current

559 results suggest the link between learning and control is reflected in the earliest time

560 period that neural control policies contribute to corrective responses. We are

561 pursuing whether and under what circumstances spinal reflexes may facilitate

562 robust control strategies.

563 The match between our model simulations and human EMG was not perfect.

564 Our model is a linearized version of the arm's biomechanics driven by actuators

565 that can produce flexor and extensor control torques. The control torques were a

566 low-pass filtered version of the control signals obtained from stochastic and robust

567 optimal feedback policies. The models did not consider the length- or velocity-

568 dependent properties of muscle ${ }^{77,78}$, nor did they consider the role of biarticular or

569 antagonist muscles. Furthermore, we did not fit the models to participant data but

570 set the parameters to examine the principle that uncertain or inaccurate internal

571 models can influence the control strategies that participants select to interact with

572 their environment. Despite being relatively simple, the models qualitatively captured

573 features of human sensorimotor control and adaptation.

574 We used a performance criterion to categorize individuals as good or partial

575 adapters. However, we really view this as a continuum where individuals may

576 tradeoff the robustness and efficiency of movement. This continuum became clear

577 when viewing how changes in muscle responses varied with the amount of 
578 adaptation. While some overlap between the behaviour of good and partial

579 adapters may reflect that robust control is capable of similar performance with

580 slightly lower levels of adaptation, some is also due to our simple performance

581 criterion (i.e., statistical contrasts misclassified some participants). Nevertheless,

582 this approach allowed us to test model predictions and capture the most

583 pronounced traits of what is indeed a continuum of strategies.

584 The continuum of strategies reveals a link between the control and adaptation

585 patterns of the dominant arm. It may also reveal an interesting parallel with work on

586 inter-limb differences in reaching movements ${ }^{79-85}$. Past work suggests the

587 dominant arm may (on average) be better suited for rapid learning of internal

588 models of predictable dynamics, while the non-dominant arm may rely on non-

589 specific control strategies that are less sensitive to unpredictable changes in

590 dynamics. If the nervous system indeed uses model-based strategies to control the

591 dominant arm, then we expect it may also display efficient corrective responses that

592 more closely resemble stochastic optimal control. Conversely, the non-dominant

593 arm may display upregulated feedback responses that more closely resemble

594 robust control. Less clear is whether differences in control persist when the arms

595 attain similar levels of performance in unpredictable dynamical environments ${ }^{86}$.

596 Our results may have implications for understanding impairments that

597 accompany neurologic disorders. Several studies have highlighted the

598 cerebellum's role in internal models for motor control and learning ${ }^{33,87-90}$. Cerebellar

599 damage can impair internal models for controlling movements ${ }^{91-95}$ and reduce the

600 rate and amount of adaptation when exposed to novel loads ${ }^{31,96-101}$. An interesting 
601 question is whether individuals with cerebellar damage would also select a robust

602 strategy when their internal models are inaccurate. There is also considerable

603 variation in the recovery of sensory and motor function after stroke ${ }^{102}$. Simulations

604 of the reaching movements of hemiparetic stroke survivors suggest impairment

605 may arise from the inability to accurately represent properties of the $\mathrm{arm}^{20}$. Stroke

606 survivors may select a robust strategy to downplay the effects of errors in the

607 internal model of their stroke-affected limb-if sensory processing is left intact.

608 However, when stroke impairs feedback and internal models, stochastic optimal

609 and robust control strategies would both be substantially impaired, leading to

610 deficits in the control and adaptation of arm movements.

611 Selecting a control strategy that is appropriate for the task and environment

612 may be an important factor in motor performance. When movement conditions are

613 highly predictable, such as a pitcher throwing a baseball, relying on an internal

614 model may reduce the variability and energetic cost of movement. In contrast,

615 robust feedback control may improve performance when dealing with unpredictable

616 disturbances while walking down a crowded street or wrestling an opponent. At the

617 extreme, it may be favourable to switch between strategies depending on the

618 situation, similar to the change in behaviour displayed by good adapters when the

619 interaction dynamics changed frequently and made it difficult to adapt. Robust

620 control may improve performance if an opponent is close when shooting a

621 basketball, whereas reducing neural feedback gains and leveraging internal models

622 may be desirable for a free throw when there is little concern of being disturbed. 


\section{Materials and Methods}

\section{Participants}

625 Forty healthy right-handed adults participated in Experiment 1 (17 males, 21-

62644 years old). The Queen's University Research Ethics Committee approved the

627 experimental procedures. Thirty healthy right-handed adults participated in

628 Experiment 2 (20 males, 20-37 years old). The protocol for Experiment 2 was

629 approved by the University of Calgary Conjoint Health and Research Ethics Board.

630 Participants gave written informed consent before participating and received

631 monetary compensation for their time. Experiments were completed in 90 minutes,

632 including participant preparation time.

633 Apparatus and Behavioural Task

634 Participants made reaching movements while seated with their right arm 635 supported in a robotic exoskeleton that can apply selective mechanical loads to the 636 shoulder and/or elbow joints (KINARM, BKIN Technologies) ${ }^{24,103}$. Targets and

637 hand-position feedback (white cursor, diameter $=0.8 \mathrm{~cm}$ ) were projected into the 638 plane of the participant's arm using a semi-silvered mirror. Direct vision of the arm 639 and hand were occluded throughout the experiment.

640 The behavioural task is shown in Figure $1 A$. Participants began the trial by 641 moving their hand to the start target $(0.625 \mathrm{~cm}$ radius $)$ and briefly holding this 642 position (1000 $\pm 500 \mathrm{~ms}$, uniformly distributed). One of three potential targets was 643 then shown in random order (T1-T3; $1 \mathrm{~cm}$ radius). We instructed participants to 644 reach the target within 450-650ms. Post-trial feedback about movement time was 645 provided during a 1s hold period at the spatial goal. The target turned green if the 
646 reach was completed on time and turned blue (too slow) or red (too fast) if the

647 participant did not reach the target within this time window. Movement times were

648 calculated as the time between when the participant's hand left the start position

649 and entered the spatial goal. An elbow extensor background load (-1 Nm; $500 \mathrm{~ms}$

650 sigmoidal ramp-up) was applied at the start of each trial. The background load

651 ramped down after movement completion (500ms sigmoidal ramp-down) and

652 remained off for $2 \mathrm{~s}$ between trials.

\section{Experiment 1. Learning to move in the presence of novel interaction loads} were proportional to the angular velocity of the elbow. The shoulder loads were applied at each time sample $(1 \mathrm{~ms})$ according to the following equation:

658

$$
\left[\begin{array}{c}
L_{s} \\
L_{e}
\end{array}\right]=\left[\begin{array}{cc}
0 & -1.5 \\
0 & 0
\end{array}\right]\left[\begin{array}{c}
\dot{\theta}_{S} \\
\dot{\theta}_{e}
\end{array}\right]
$$

where $\left[\begin{array}{ll}L_{s} & L_{e}\end{array}\right]^{\mathrm{T}}$ represent interaction loads $(\mathrm{Nm})$ applied by the robotic device, and

$660 \dot{\theta}_{s}$ and $\dot{\theta}_{e}$ represent the angular velocities of the shoulder and elbow (rad/s). Note

661 the shoulder loads were applied in the direction opposite the elbow's angular 662 velocity, such that elbow flexion produced shoulder extensor loads.

663 The experiment was divided into 15 blocks ( 4-5 min/block) separated into three

664 phases: baseline (4 blocks), early adaptation ( 5 blocks), and late adaptation (6 665 blocks). Each block consisted of 44 trials, including 20 movements to T1, 12 666 movements to T2, and 12 movements to T3. Four of the 20 movements to T1 were 667 interleaved step-torque perturbations. Two-minute rest breaks were provided after 668 every 4 blocks. Additional rest breaks were provided at the participant's request. 
669 Our paradigm uses training targets that require combined shoulder and elbow

670 motion (T2; $10^{\circ}$ shoulder flexion, $10^{\circ}$ elbow flexion), or only elbow motion (T3, $20^{\circ}$

671 elbow flexion). Participants also reached to a separate test target that required only

672 shoulder flexion motion ( $\mathrm{T} 1 ; 20^{\circ}$ shoulder flexion). Elbow motion while reaching $\mathrm{T} 1$

673 was limited $\left(<2^{\circ}\right)$ and produced only small residual loads while participants reached

674 to the test target (integrated shoulder load at T1 $<3 \%$ of integrated shoulder loads

675 experienced at T2 and T3). This allowed us to maintain relatively similar amounts

676 of muscle activity while participants reached T1. Our design allowed us to test if

677 knowledge of the interaction loads expressed while reaching the training targets

678 (T2, T3) was reflected in shoulder muscle responses when disturbed by a step-

679 torque perturbation while reaching to the test target (T1).

680

\section{Feedback Responses to Mechanical Perturbations}

Feedback responses were measured on random trials by applying step-torque

683 perturbations that extended the elbow (-2 Nm at shoulder and elbow; 10ms

684 sigmoidal rise-time) while participants reached the test target. The step-torque

685 perturbations were triggered the instant the participant's hand left the start position.

686 Equal amounts of torque were applied at the shoulder and elbow joints ${ }^{44}$.

687 Participants were instructed to reach the spatial goal within the same time window 688 as unperturbed trials and received the same post-trial feedback. 
692 Experiment 2. Interacting with rapidly changing interaction dynamics

693 Experiment 2 used the same behavioural task as Experiment 1. Participants

$694(\mathrm{n}=30)$ first performed unloaded movements. We then introduced the same

695 interaction loads at the shoulder (load constant $=-1.5 \mathrm{Nm} . \mathrm{s} / \mathrm{rad}$ ). After participants

696 completed the adaptation phase, we varied the load coefficient in blocks of 11 trials.

697 Each block included an interleaved step-torque perturbation that displaced the 698 elbow into extension while participants reached to the test target (T1). The size of

699 the load coefficient was altered in each block of trials while maintaining the same 700 average coefficient as when exposed to constant interaction dynamics. Other 701 aspects of the experiment were identical to Experiment 1.

702

703 Data Analysis

704 Kinematic recordings and learning analysis.

705 Shoulder and elbow joint kinematics were sampled at $1 \mathrm{kHz}$ and digitally low-

706 pass filtered ( $2^{\text {nd }}$-order, dual-pass Butterworth, $30 \mathrm{~Hz}$ effective cutoff). We

707 calculated the perpendicular distance between the hand and a straight line joining

708 the start position and goal at each time sample ${ }^{104}$. We measured changes in

709 reaching patterns by comparing peak hand-path deviations in the baseline block

710 with the last 25 reaching movements to each target in the adaptation block.

711 Participants were classified as good adapters if their hand paths returned to near

712 baseline levels of performance at the end of adaptation (single-sided paired t-test

713 for individual participants, $p>0.05$ ), or partial adapters if their hand paths still

714 deviated from their baseline movements at the end of adaptation (single-sided t- 
715 test for individual participants, $p<0.05)$. The amount of learning (\% adaptation) was

716 determined by comparing hand-path deviations at the end of learning with baseline

717 movements:

$718 \%$ adaptation $=\left(1-\left(\frac{\text { perp.deviation }_{\text {adapt }}-\text { perp.deviation }_{\text {baseline }}}{\text { perp.deviation }_{\text {baseline }}}\right)\right) * 100 \%$.

719 The hand velocity profile and peak velocities in the direction of the target

720 were computed on a trial-by-trial basis for each participant. Averages were then

721 compared across groups of good and partial adapters (single-sided unpaired t-test,

$722 p<0.05)$. We then normalized the hand-path variability displayed by partial adapters

723 during movements to each separate target (T1-T3) to the average tangential hand-

724 path variability displayed by good adapters while reaching the same target. We

725 contrasted the normalized hand-path variability between good and partial adapters

726 (single-sided unpaired t-test, $p<0.05$ ).

727

728

EMG Recordings and Analysis

729 EMG of the upper limb muscles was recorded using bipolar surface electrodes

730 (DE 2.1 Single Differential Electrode, Delsys). EMG signals were amplified online

731 (gain $=10^{3}-10^{4}$ ) and sampled at $1-\mathrm{kHz}$. We recorded the activity of the

732 monoarticular elbow muscles (brachioradialis, elbow flexor; triceps lateralis, elbow

733 extensor) and shoulder muscles (pectoralis major, shoulder flexor; posterior deltoid,

734 shoulder extensor). We also recorded from the biarticular flexor muscles (biceps

735 brachii, short head) and extensor muscles (triceps longus). We cleaned the skin

736 with alcohol before attaching electrodes over each muscle. A ground electrode was

737 placed over the patella or elbow. 
738 EMG data were aligned to perturbation onset, band-pass filtered (3 ${ }^{\text {rd }}$-order, 20-

$739450 \mathrm{~Hz}$ pass band, dual-pass Butterworth) and full-wave rectified. We normalized

740 each muscle's rectified activity during reaching to its activity while maintaining

741 postural control at the start position against $1 \mathrm{Nm}$ extensor (brachioradialis, biceps

742 brachii, pectoralis major) or flexor background loads (triceps lateralis, triceps

743 longus, posterior deltoid). The posture task was completed at the start of the

744 experiment (five 15s trials for each load direction). Additional details about the EMG

745 normalization can be found in Cluff and Scott $(2013)^{72}$.

746 Muscle stretch responses were aligned to the onset of the step-torque

747 perturbation. We subtracted the average EMG in unperturbed trials from perturbed

748 trials $(\triangle E M G)$. We then calculated each muscle's average activity $(\triangle E M G)$ in

749 predefined time windows on a trial-by-trial basis for each participant ${ }^{72,105}$ : pre-

750 perturbation (PRE: -50-0 ms), short-latency (SL: 25-50 ms), long-latency (LL: 50-

$751105 \mathrm{~ms}$ ) and early voluntary (VOL: 105-180 ms). We examined how good and

752 partial adapters altered their muscle responses by comparing average EMG

753 responses to the step-torque perturbation in each time window between the

754 baseline and late adaptation phases (paired t-tests). Similarly, we compared hand

755 motion caused by the step-torque perturbation between baseline and late

756 adaptation trials. EMG data were smoothed with a bidirectional 5-ms moving

757 average filter for display purposes. All statistical tests were performed on

758 unsmoothed EMG data.

759 


\section{Optimal Feedback Control Models}

762 The control models are based on a linear approximation of the arm moving

763 in the horizontal plane. We calculated the inertia of the arm segments based on the

764 physical characteristics of our human participants using standard anthropometric

765 estimates ${ }^{106}$. We combined these inertial estimates with the inertial properties of

766 the robotic exoskeleton. The mechanical model of the arm was coupled with a first-

767 order, low-pass filter linking control input to muscle force to approximate the

768 dynamics of muscular contraction (time constant: $60 \mathrm{~ms}$ ) ${ }^{107}$. We simulated reaching

769 movements to the same targets (T1-T3), with the same time $(450-650 \mathrm{~ms})$ and

770 accuracy demands (target diameter $=2 \mathrm{~cm}$ ) as our human experiments.

771 We consider two optimal control algorithms. The first follows the literature on

772 sensorimotor control and learning, and is based on stochastic optimal control, or

773 linear-quadratic-Gaussian regulator (LQG control) $)^{108}$. This control model assumes

774 plant uncertainty is captured by a random variable that follows a known (Gaussian)

775 distribution. The second control algorithm is based on robust control, formulated

776 here in the context of $H_{\infty}$-optimal control. The robust control algorithm achieves

777 optimal attenuation of unknown external disturbances, including uncertainty or

778 errors in the internal model of the plant (i.e., the upper $\operatorname{limb})^{40}$. This control model

779 has recently been used to describe human sensorimotor control ${ }^{109}$ and can be

780 designed to handle errors or uncertainty in the internal model of the arm, including

781 the error that we induce experimentally by introducing the novel interaction load.

782 The discrete time control system is expressed in the following equation, in

783 which $x_{t}$ represents the state vector including joint angles, velocities and torques: 
$x_{t+1}=(A+\Delta A) x_{t}+(B+\Delta B) u_{t}+\xi_{t}$.

786

787 The matrices $A$ and $B$ are the modeled (or expected) dynamics, $\Delta A$ and $\Delta B$ are the

788 model errors, and $\xi_{t}$ is a zero-mean Gaussian disturbance with covariance $\Sigma_{\xi}$. The

789 discrete time system is coupled with the feedback equation:

790

$791 y_{t}=x_{t-h}+\zeta_{t}$,

792

793 where $h$ is the feedback delay expressed in number of time samples and $\zeta_{t}$ is the

794 additive Gaussian noise that corrupts sensory feedback with covariance $\Sigma_{\zeta}$.

795 Temporal delays were handled with standard system augmentation ${ }^{60}$. Equation 1

796 can be transformed into:

797

798

$x_{t+1}=A x_{t}+B u_{t}+w_{t}$,

799

800 where the term $w_{t}:=\Delta A x_{t}+\Delta B u_{t}+\xi_{t}$ captures the noise disturbances, as well as

801 un-modeled limb dynamics. With these definitions, the control problem consists of

802 minimizing the following cost-function ${ }^{40}$ :

803

$804 J(x, u, w)=\sum_{t=1}^{N} x_{t}^{T} Q_{t} x_{t}+u_{t}^{T} u_{t}-\gamma^{2} w_{t}^{T} w_{t}$

805 while using the incomplete state information from Equation 2. 
Stochastic optimal control and robust control solve this problem in different ways. In the framework of stochastic optimal control, it is assumed that $\Delta A=\Delta B=$

8080 , and the control solution minimizes the expected value of $J(x, u, w)$. A well-known 809 solution is given by (extended) LQG control ${ }^{38}$. The robust control approach 810 considers that $\Delta A$ and $\Delta B$ can be non-zero, and the controller minimizes the impact

811 of this unknown disturbance. The control law is derived in the worst-case scenario

812 formulated as a zero-sum dynamic game. The full derivation of the robust controller 813 was provided in detail by Basar and Bernhard ${ }^{40}$. Observe that in our experiment, 814 the internal model error following the introduction of the novel load applies to the 815 state variables by mapping elbow velocity into shoulder torque. As a consequence, 816 our experiment corresponds to the situation where $\Delta B=0$ and $\Delta A \neq 0$.

817 The two controllers are coupled with a state estimator, which is necessary to 818 handle noise in sensory signals as well as temporal delays induced by the 819 transmission and processing of neural signals (50ms delay, Equation 2). The 820 optimal state estimator for the stochastic optimal controller is the standard Kalman 821 filter ${ }^{76}$. The optimal estimator for the robust controller differs from the Kalman filter 822 because the plant uncertainty also affects how feedback signals are used to correct 823 the prediction about the present state of the limb. However, the general form of this 824 estimator is similar to the Kalman filter in the sense that it is a linear combination of 825 the previous state estimate, control input and feedback vector ${ }^{40}$.

826 The cost matrices $\left(Q_{t}\right)$ penalize all state variables to ensure the robust 827 control problem is not ill-posed ${ }^{3}$. We applied a constant cost on velocity and muscle 828 force (numerical values: $30 \times(\text { velocity })^{2}$ and $\left.0.1 \times(\text { torque })^{2}\right)$, whereas the 
829 running cost on joint angles followed a quadratic build up towards the final cost

830 (final cost: $\left.6 \times 10^{3} \times(\text { position error })^{2}\right)$. These matrices were adjusted to ensure

831 the robust control problem is well defined (i.e., the cost matrix $Q_{t}$ is invertible) ${ }^{40}$, as

832 well as to generate movement trajectories that qualitatively resembled the

833 behaviour of our experimental participants. The cost parameters were kept identical

834 for the two control designs, and thus the changes in behavior indeed reflect

835 differences in the control strategies. The parameter $\gamma$ is jointly optimized with the

836 control function and represents the optimal level of disturbance attenuation. The

837 two controllers converge to a linear control law applied to the estimated state. Table

8381 lists the model parameters and provides numerical values used in the simulations.

839 Paralleling the experimental procedures, we first characterized reaching

840 movements during a baseline period consisting of unloaded movements $(\Delta A=0)$.

841 Step-torque perturbations were simulated with abrupt changes in the external

842 torque applied during the simulation runs ${ }^{39,60}$.

843 We then examined how the models performed when reaching to the same

844 targets in the presence of a novel interaction load $(\Delta A \neq 0)$ that was identical to our

845 human experiments and was proportional to the instantaneous angular velocity of

846 the elbow. We simulated learning by manipulating knowledge of the interaction

847 load. We varied knowledge of the parameter, $L$ (-1.5 Nm.s/rad), by multiplying with

848 a scalar $(0,0.25,0.50,0.75,1.00)$, which is the constant that maps elbow angular

849 velocities onto interaction loads applied at the shoulder (corresponding to 0, 10, 25,

85050,75 , and $100 \%$ knowledge of the load coefficient). For comparison purposes, we

851 constrained knowledge of the novel load to the amount of learning displayed by 
852 good (94\% adaptation) and partial adapters (72\% adaptation). We also looked at

853 how the two control algorithms respond to the same step-torque perturbations

854 before (25\% learning) and after adapting to novel interaction loads (robust control:

855 72\% learning; LQG control: 94\% learning). Finally, we calculated tangential hand

856 velocity profiles for the models, as well as hand-path variability during movement ( $t$

$857=0-800 \mathrm{~ms}$ ) for the LQG and robust control algorithms. We then expressed hand-

858 path variability for the robust controller as a percentage of that noted for the LQG

859 controller (i.e., 1 = identical hand-path variability for both controllers).

860 Note the structure of the cost function was partially constrained by the

861 requirements of the game-theoretic approach used for the robust controller (i.e.,

$862 Q_{t}$ must be invertible). Importantly, this approach allowed us to use the same cost

863 function structure for simulations with the robust and LQG controllers. Thus, the

864 free parameters were the amount of noise corrupting the motor command (process

865 noise) and sensory feedback (observation noise). We reduced the number of free

866 parameters by assuming sensory and motor noise terms were equivalent.

867 Changing the cost function and noise parameters will alter the quantitative

868 correspondence between model simulations and human behavior. Although human

869 behavior may not be captured quantitatively by other parameter settings, the

870 fundamental principle that robust control reduces sensitivity to internal model

871 errors, and the trade-off between efficiency and robustness remains true across all

872 parameter settings. Thus, the difference in how robust and LQG-control solve the

873 control problem has an overarching impact on how the controllers respond to

874 external perturbations and novel interaction loads. 
875 Author contributions: T.C. performed the experiments; T.C., F.C., S.H.S.

876 analyzed the data, F.C. performed the simulations, T.C., F.C., S.HS. wrote the

877 paper.

878

879 Code and data availability: Custom computer code was used to control the 880 experimental task, analyze behavioural data and perform the computational 881 modeling. Computer code and behavioral data are available upon request to the 882 corresponding author.

883

884 Competing financial interests: Stephen $\mathrm{H}$. Scott is the co-founder of BKIN

885 Technologies, which commercializes the robotic device used in our study.

Acknowledgements: We thank Kim Moore and Justin Peterson for their technical

889 support. We thank members of the LIMB Lab for their helpful feedback and comments. We would also like to thank Julien Hendrickx for critical comments on

891 an earlier version of this manuscript. This work was supported by NSERC

892 Discovery grants to T.C. and S.H.S. 
893

894

895

896

897

898

899

900

901

902

903

904

905

906

907

908

909

910

911

912

913

914

915

916

917

918

919

920

921

922

923

924

925

926

927

928

929

930

931

932

933

934

935

936

937

938

939

940

\section{References}

1. Cassidy, T. G., Jones, R. L. \& Potrac, P. Understanding Sports Coaching: The Social, Cultural and Pedagogical Foundations of Coaching Practice. (Routledge, 2008).

2. Yarrow, K., Brown, P. \& Krakauer, J. W. Inside the brain of an elite athlete: the neural processes that support high achievement in sports. Nat. Rev. Neurosci. 10, 585-596 (2009).

3. Zatorre, R. J., Fields, R. D. \& Johansen-Berg, H. Plasticity in gray and white: neuroimaging changes in brain structure during learning. Nat. Neurosci. 15, 528536 (2012).

4. Zatorre, R. J., Chen, J. L. \& Penhune, V. B. When the brain plays music: auditory-motor interactions in music perception and production. Nat. Rev. Neurosci. 8, 547-558 (2007).

5. Doyon, J. et al. Contributions of the basal ganglia and functionally related brain structures to motor learning. Behav. Brain Res. 199, 61-75 (2009).

6. Wolpert, D. M. \& Flanagan, J. R. Computations underlying sensorimotor learning. Curr. Opin. Neurobiol. 37, 7-11 (2016).

7. Wolpert, D. M., Diedrichsen, J. \& Flanagan, J. R. Principles of sensorimotor learning. Nat. Rev. Neurosci. 12, 739-751 (2011).

8. Shadmehr, R., Smith, M. A. \& Krakauer, J. W. Error Correction, Sensory Prediction, and Adaptation in Motor Control. Annu. Rev. Neurosci. 33, 89-108 (2010).

9. Kiper, P. et al. Computational models and motor learning paradigms: Could they provide insights for neuroplasticity after stroke? An overview. J. Neurol. Sci. 369, 141-148 (2016).

10. Seidler, R. D. Multiple Motor Learning Experiences Enhance Motor Adaptability. J. Cogn. Neurosci. 16, 65-73 (2004).

11. Wu, H. G., Miyamoto, Y. R., Castro, L. N. G., Ölveczky, B. P. \& Smith, M. A. Temporal structure of motor variability is dynamically regulated and predicts motor learning ability. Nat. Neurosci. 17, 312-321 (2014).

12. Albert, S. T. \& Shadmehr, R. The Neural Feedback Response to Error As a Teaching Signal for the Motor Learning System. J. Neurosci. 36, 4832-4845 (2016).

13. Anguera, J. A., Reuter-Lorenz, P. A., Willingham, D. T. \& Seidler, R. D. Failure to Engage Spatial Working Memory Contributes to Age-related Declines in Visuomotor Learning. J. Cogn. Neurosci. 23, 11-25 (2010).

14. Christou, A. I., Miall, R. C., McNab, F. \& Galea, J. M. Individual differences in explicit and implicit visuomotor learning and working memory capacity. Sci. Rep. 6, 36633 (2016).

15. Ruitenberg, M. F. L. et al. Neural correlates of multi-day learning and savings in sensorimotor adaptation. Sci. Rep. 8, 14286 (2018).

16. Scheidt, R. A., Reinkensmeyer, D. J., Conditt, M. A., Rymer, W. Z. \& MussaIvaldi, F. A. Persistence of Motor Adaptation During Constrained, Multi-Joint, Arm Movements. J. Neurophysiol. 84, 853-862 (2000).

17. Smith, M. A., Ghazizadeh, A. \& Shadmehr, R. Interacting Adaptive Processes with Different Timescales Underlie Short-Term Motor Learning. PLOS Biol 4, e179 (2006). 
941

942

943

944

945

946

947

948

949

950

951

952

953

954

955

956

957

958

959

960

961

962

963

964

965

966

967

968

969

970

971

972

973

974

975

976

977

978

979

980

981

982

983

984

985

986

987

988

18. Sing, G. C. \& Smith, M. A. Reduction in Learning Rates Associated with Anterograde Interference Results from Interactions between Different Timescales in Motor Adaptation. PLOS Comput. Biol. 6, e1000893 (2010).

19. Howard, I. S., Ingram, J. N., Franklin, D. W. \& Wolpert, D. M. Gone in 0.6 Seconds: The Encoding of Motor Memories Depends on Recent Sensorimotor States. J. Neurosci. 32, 12756-12768 (2012).

20. Sketch, S. M., Simpson, C. S., Crevecoeur, F. \& Okamura, A. M. Simulating the impact of sensorimotor deficits on reaching performance. in 2017 International Conference on Rehabilitation Robotics (ICORR) 31-37 (2017). doi:10.1109/ICORR.2017.8009217

21. Miall, R. C., Weir, D. J., Wolpert, D. M. \& Stein, J. F. Is the Cerebellum a Smith Predictor? J. Mot. Behav. 25, 203-216 (1993).

22. Gonzalez Castro, L. N., Hadjiosif, A. M., Hemphill, M. A. \& Smith, M. A. Environmental Consistency Determines the Rate of Motor Adaptation. Curr. Biol. 24, 1050-1061 (2014).

23. Franklin, S., Wolpert, D. M. \& Franklin, D. W. Rapid visuomotor feedback gains are tuned to the task dynamics. J. Neurophysiol. jn.00748.2016 (2017). doi:10.1152/jn.00748.2016

24. Singh, K. \& Scott, S. H. A motor learning strategy reflects neural circuitry for limb control. Nat. Neurosci. 6, 399-403 (2003).

25. Cluff, T. \& Scott, S. H. Rapid Feedback Responses Correlate with Reach Adaptation and Properties of Novel Upper Limb Loads. J. Neurosci. 33, 1590315914 (2013).

26. Pruszynski, J. A., Kurtzer, I., Lillicrap, T. P. \& Scott, S. H. Temporal Evolution of "Automatic Gain-Scaling". J. Neurophysiol. 102, 992-1003 (2009).

27. Bedingham, W. \& Tatton, W. G. Dependence of EMG Responses Evoked by Imposed Wrist Displacements on Pre-existing Activity in the Stretched Muscles. Can. J. Neurol. Sci. 11, 272-280 (1984).

28. Stein, R. B., Hunter, I. W., Lafontaine, S. R. \& Jones, L. A. Analysis of shortlatency reflexes in human elbow flexor muscles. J. Neurophysiol. 73, 1900-1911 (1995).

29. Matthews, P. B. Observations on the automatic compensation of reflex gain on varying the pre-existing level of motor discharge in man. J. Physiol. 374, 73-90 (1986).

30. Shadmehr, R. \& Moussavi, Z. M. K. Spatial Generalization from Learning Dynamics of Reaching Movements. J. Neurosci. 20, 7807-7815 (2000).

31. Criscimagna-Hemminger, S. E., Bastian, A. J. \& Shadmehr, R. Size of Error Affects Cerebellar Contributions to Motor Learning. J. Neurophysiol. 103, 22752284 (2010).

32. Shadmehr, R. \& Mussa-Ivaldi, F. A. Adaptive representation of dynamics during learning of a motor task. J. Neurosci. 14, 3208-3224 (1994).

33. Miall, R. C. \& Wolpert, D. M. Forward Models for Physiological Motor Control. Neural Netw. 9, 1265-1279 (1996).

34. Franklin, S., Wolpert, D. M. \& Franklin, D. W. Visuomotor feedback gains upregulate during the learning of novel dynamics. J. Neurophysiol. 108, 467-478 (2012).

35. Gribble, P. L. \& Ostry, D. J. Compensation for Interaction Torques During Singleand Multijoint Limb Movement. J. Neurophysiol. 82, 2310-2326 (1999). 
989

990

991

992

993

994

995

996

997

998

999

1000

1001

1002

1003

1004

1005

1006

1007

1008

1009

1010

1011

1012

1013

1014

1015

1016

1017

1018

1019

1020

1021

1022

1023

1024

1025

1026

1027

1028

1029

1030

1031

1032

1033

1034

1035

1036
36. Maeda, R. S., Cluff, T., Gribble, P. L. \& Pruszynski, J. A. Compensating for intersegmental dynamics across the shoulder, elbow, and wrist joints during feedforward and feedback control. J. Neurophysiol. 118, 1984-1997 (2017).

37. Maeda, R. S., Cluff, T., Gribble, P. L. \& Pruszynski, J. A. Feedforward and Feedback Control Share an Internal Model of the Arm's Dynamics. J. Neurosci. 38, 10505-10514 (2018).

38. Todorov, E. Stochastic Optimal Control and Estimation Methods Adapted to the Noise Characteristics of the Sensorimotor System. Neural Comput. 17, 10841108 (2005).

39. Crevecoeur, F. \& Scott, S. H. Beyond Muscles Stiffness: Importance of StateEstimation to Account for Very Fast Motor Corrections. PLOS Comput Biol 10, e1003869 (2014).

40. Başar, T. \& Bernhard, P. H-Infinity Optimal Control and Related Minimax Design Problems: A Dynamic Game Approach. (Springer Science \& Business Media, 2008).

41. Zhou, K., Doyle, J. C. \& Glover, K. Robust and Optimal Control. Automatica 11, 2095 (1997).

42. Brown, S. H. \& Cooke, J. D. Amplitude- and instruction-dependent modulation of movement-related electromyogram activity in humans. J. Physiol. 316, 97-107 (1981).

43. Kurtzer, I., Pruszynski, J. A. \& Scott, S. H. Long-Latency Responses During Reaching Account for the Mechanical Interaction Between the Shoulder and Elbow Joints. J. Neurophysiol. 102, 3004-3015 (2009).

44. Hadjiosif, A. M. \& Smith, M. A. Flexible Control of Safety Margins for Action Based on Environmental Variability. J. Neurosci. 35, 9106-9121 (2015).

45. Seidler, R. D., Mulavara, A. P., Bloomberg, J. J. \& Peters, B. T. Individual predictors of sensorimotor adaptability. Front. Syst. Neurosci. 9, (2015).

46. Riding, R. \& Rayner, S. Cognitive Styles and Learning Strategies: Understanding Style Differences in Learning and Behavior. (Routledge, 2013).

47. Riding, R. J. \& Sadler-Smith, E. Cognitive Style and Learning Strategies: Some Implications for Training Design. Int. J. Train. Dev. 1, 199-208 (1997).

48. Schmeck, R. R. Learning Strategies and Learning Styles. (Springer Science \& Business Media, 2013).

49. Entwistle, N. \& Ramsden, P. Understanding Student Learning (Routledge Revivals). (Routledge, 2015).

50. Sugrue, L. P., Corrado, G. S. \& Newsome, W. T. Choosing the greater of two goods: neural currencies for valuation and decision making. Nat. Rev. Neurosci. 6, 363-375 (2005).

51. Lee, D. Game theory and neural basis of social decision making. Nat. Neurosci. 11, 404-409 (2008).

52. Palidis, D. J., Cashaback, J. \& Gribble, P. Distinct Neural Signatures of Reward and Sensory Prediction Error in Motor Learning. bioRxiv 262576 (2018). doi:10.1101/262576

53. Izawa, J., Rane, T., Donchin, O. \& Shadmehr, R. Motor Adaptation as a Process of Reoptimization. J. Neurosci. 28, 2883-2891 (2008).

54. Takahashi, C. D., Scheidt, R. A. \& Reinkensmeyer, D. J. Impedance Control and Internal Model Formation When Reaching in a Randomly Varying Dynamical Environment. J. Neurophysiol. 86, 1047-1051 (2001). 
1037

1038

1039

1040

1041

1042

1043

1044

1045

1046

1047

1048

1049

1050

1051

1052

1053

1054

1055

1056

1057

1058

1059

1060

1061

1062

1063

1064

1065

1066

1067

1068

1069

1070

1071

1072

1073

1074

1075

1076

1077

1078

1079

1080

1081

1082

1083

1084

1085

55. Wei, K., Wert, D. \& Körding, K. The Nervous System Uses Nonspecific Motor Learning in Response to Random Perturbations of Varying Nature. J. Neurophysiol. 104, 3053-3063 (2010).

56. Körding, K. P. \& Wolpert, D. M. Bayesian integration in sensorimotor learning. Nature 427, 244-247 (2004).

57. Wei, K. \& Körding, K. Uncertainty of Feedback and State Estimation Determines the Speed of Motor Adaptation. Front. Comput. Neurosci. 4, (2010).

58. Berniker, M., Voss, M. \& Kording, K. Learning Priors for Bayesian Computations in the Nervous System. PLOS ONE 5, e12686 (2010).

59. Horak, F. B. \& Diener, H. C. Cerebellar control of postural scaling and central set in stance. J. Neurophysiol. 72, 479-493 (1994).

60. Crevecoeur, F. \& Scott, S. H. Priors Engaged in Long-Latency Responses to Mechanical Perturbations Suggest a Rapid Update in State Estimation. PLOS Comput. Biol. 9, e1003177 (2013).

61. Welch, T. D. J. \& Ting, L. H. Mechanisms of Motor Adaptation in Reactive Balance Control. PLOS ONE 9, e96440 (2014).

62. Berniker, M. \& Kording, K. Estimating the sources of motor errors for adaptation and generalization. Nat. Neurosci. 11, 1454-1461 (2008).

63. Nezafat, R., Shadmehr, R. \& Holcomb, H. H. Long-term adaptation to dynamics of reaching movements: a PET study. Exp. Brain Res. 140, 66-76 (2001).

64. Stark-Inbar, A., Raza, M., Taylor, J. A. \& Ivry, R. B. Individual differences in implicit motor learning: task specificity in sensorimotor adaptation and sequence learning. J. Neurophysiol. 117, 412-428 (2017).

65. Doyon, J. \& Benali, H. Reorganization and plasticity in the adult brain during learning of motor skills. Curr. Opin. Neurobiol. 15, 161-167 (2005).

66. Dimitriou, M. Task-dependent modulation of spinal and transcortical stretch reflexes linked to motor learning rate. Behav. Neurosci. 132, 194-209 (2018).

67. Burdet, E., Osu, R., Franklin, D. W., Milner, T. E. \& Kawato, M. The central nervous system stabilizes unstable dynamics by learning optimal impedance. Nature 414, 446 (2001).

68. Franklin, D. W. et al. CNS Learns Stable, Accurate, and Efficient Movements Using a Simple Algorithm. J. Neurosci. 28, 11165-11173 (2008).

69. Franklin, D. W. et al. Endpoint Stiffness of the Arm Is Directionally Tuned to Instability in the Environment. J. Neurosci. 27, 7705-7716 (2007).

70. Franklin, D. W. \& Wolpert, D. M. Computational Mechanisms of Sensorimotor Control. Neuron 72, 425-442 (2011).

71. Burdet, E. et al. A method for measuring endpoint stiffness during multi-joint arm movements. J. Biomech. 33, 1705-1709 (2000).

72. Cluff, T. \& Scott, S. H. Rapid Feedback Responses Correlate with Reach Adaptation and Properties of Novel Upper Limb Loads. J. Neurosci. 33, 1590315914 (2013).

73. Scott, S. H. Optimal feedback control and the neural basis of volitional motor control. Nat. Rev. Neurosci. 5, 532-546 (2004).

74. Pruszynski, J. A. \& Scott, S. H. Optimal feedback control and the long-latency stretch response. Exp. Brain Res. 218, 341-359 (2012).

75. Scott, S. H. The computational and neural basis of voluntary motor control and planning. Trends Cogn. Sci. 16, 541-549 (2012).

76. Scott, S. H. A Functional Taxonomy of Bottom-Up Sensory Feedback Processing for Motor Actions. Trends Neurosci. 39, 512-526 (2016). 
1086

1087

1088

1089

1090

1091

1092

1093

1094

1095

1096

1097

1098

1099

1100

1101

1102

1103

1104

1105

1106

1107

1108

1109

1110

1111

1112

1113

1114

1115

1116

1117

1118

1119

1120

1121

1122

1123

1124

1125

1126

1127

1128

1129

1130

1131

1132

1133

1134
77. Scott, S. H., Brown, I. E. \& Loeb, G. E. Mechanics of feline soleus: I. Effect of fascicle length and velocity on force output. J. Muscle Res. Cell Motil. 17, 207219 (1996).

78. Cheng, E. J., Brown, I. E. \& Loeb, G. E. Virtual muscle: a computational approach to understanding the effects of muscle properties on motor control. J. Neurosci. Methods 101, 117-130 (2000).

79. Sainburg, R. L. Evidence for a dynamic-dominance hypothesis of handedness. Exp. Brain Res. 142, 241-258 (2002).

80. Sainburg, R. L. Convergent models of handedness and brain lateralization. Front. Psychol. 5, (2014).

81. Sainburg, R. L. Laterality of Basic Motor Control Mechanisms: Different Roles of the Right and Left Brain Hemispheres. Laterality Sports Theor. Appl. 155-177 (2016). doi:10.1016/B978-0-12-801426-4.00008-0

82. Yadav, V. \& Sainburg, R. L. Motor lateralization is characterized by a serial hybrid control scheme. Neuroscience 196, 153-167 (2011).

83. Bagesteiro, L. B. \& Sainburg, R. L. Handedness: Dominant Arm Advantages in Control of Limb Dynamics. J. Neurophysiol. 88, 2408-2421 (2002).

84. Sainburg, R. L., Ghez, C., Kalakanis, D., Ghez, C. \& Dy, D. K. I. Intersegmental dynamics are controlled by sequential anticipatory error correction and postural mechanisms. J. Neurophysiol. 1045-1056 (1999).

85. Wang, J. \& Sainburg, R. L. Interlimb Transfer of Novel Inertial Dynamics Is Asymmetrical. J. Neurophysiol. 92, 349-360 (2004).

86. Yadav, V. \& Sainburg, R. L. Limb Dominance Results from Asymmetries in Predictive and Impedance Control Mechanisms. PLOS ONE 9, e93892 (2014).

87. Wolpert, D. M., Miall, R. C. \& Kawato, M. Internal models in the cerebellum. Trends Cogn. Sci. 2, 338-347 (1998).

88. Bastian, A. J. Learning to predict the future: the cerebellum adapts feedforward movement control. Curr. Opin. Neurobiol. 16, 645-649 (2006).

89. Paulin, M. G. Evolution of the cerebellum as a neuronal machine for Bayesian state estimation. J. Neural Eng. 2, S219 (2005).

90. Nowak, D. A., Topka, H., Timmann, D., Boecker, H. \& Hermsdörfer, J. The role of the cerebellum for predictive control of grasping. The Cerebellum 6, 7 (2007).

91. Bhanpuri, N. H., Okamura, A. M. \& Bastian, A. J. Predicting and correcting ataxia using a model of cerebellar function. Brain awu115 (2014).

doi:10.1093/brain/awu115

92. Kurtzer, I. et al. Cerebellar damage diminishes long-latency responses to multijoint perturbations. J. Neurophysiol. 109, 2228-2241 (2013).

93. Bastian, A. J., Martin, T. A., Keating, J. G. \& Thach, W. T. Cerebellar ataxia: abnormal control of interaction torques across multiple joints. J. Neurophysiol. 76, 492-509 (1996).

94. Topka, H., Konczak, J., Schneider, K., Boose, A. \& Dichgans, J. Multijoint arm movements in cerebellar ataxia:Abnormal control of movement dynamics. Exp. Brain Res. 119, 493-503 (1998).

95. Goodkin, H. P., Keating, J. G., Martin, T. A. \& Thach, W. T. Preserved Simple and Impaired Compound Movement After Infarction in the Territory of the Superior Cerebellar Artery. Can. J. Neurol. Sci. 20, S93-S104 (1993).

96. Izawa, J., Criscimagna-Hemminger, S. E. \& Shadmehr, R. Cerebellar Contributions to Reach Adaptation and Learning Sensory Consequences of Action. J. Neurosci. 32, 4230-4239 (2012). 
97. Smith, M. A. \& Shadmehr, R. Intact Ability to Learn Internal Models of Arm Dynamics in Huntington's Disease But Not Cerebellar Degeneration. J. Neurophysiol. 93, 2809-2821 (2005). Ataxia Progressively Impairs Force Adaptation During Goal-Directed Arm Movements. J. Neurophysiol. 91, 230-238 (2004).

99. Donchin, O. et al. Cerebellar regions involved in adaptation to force field and visuomotor perturbation. J. Neurophysiol. 107, 134-147 (2011).

100. Miall, R. C., Christensen, L. O. D., Cain, O. \& Stanley, J. Disruption of State Estimation in the Human Lateral Cerebellum. PLOS Biol. 5, e316 (2007).

101. Tseng, Y., Diedrichsen, J., Krakauer, J. W., Shadmehr, R. \& Bastian, A. J. Sensory Prediction Errors Drive Cerebellum-Dependent Adaptation of Reaching. J. Neurophysiol. 98, 54-62 (2007).

102. Semrau, J. A., Herter, T. M., Scott, S. H. \& Dukelow, S. P. Examining Differences in Patterns of Sensory and Motor Recovery After Stroke With Robotics. Stroke 46, 3459-3469 (2015).

103. Scott, S. H. Apparatus for measuring and perturbing shoulder and elbow joint positions and torques during reaching. J. Neurosci. Methods 89, 119-127 (1999).

104. Thoroughman, K. A. \& Shadmehr, R. Electromyographic Correlates of Learning an Internal Model of Reaching Movements. J. Neurosci. 19, 8573-8588 (1999).

105. Cluff, T. \& Scott, S. H. Apparent and Actual Trajectory Control Depend on the Behavioral Context in Upper Limb Motor Tasks. J. Neurosci. 35, 12465-12476 (2015).

106. Winter, D. A. Biomechanics and Motor Control of Human Movement. (John Wiley \& Sons, 2009).

107. Brown, I. E., Cheng, E. J. \& Loeb, G. E. Measured and modeled properties of mammalian skeletal muscle. II. The effectsof stimulus frequency on force-length and force-velocity relationships. J. Muscle Res. Cell Motil. 20, 627-643

108. Todorov, E. \& Jordan, M. I. Optimal feedback control as a theory of motor coordination. Nat. Neurosci. 5, 1226-1235 (2002).

109. Ueyama, Y. Mini-max feedback control as a computational theory of sensorimotor control in the presence of structural uncertainty. Front. Comput. Neurosci. 8, (2014). 


\section{Figures, Tables and Captions}

1172

1173

1174

1175

\section{Exemplar Partial Adapter Exemplar Good Adapter}

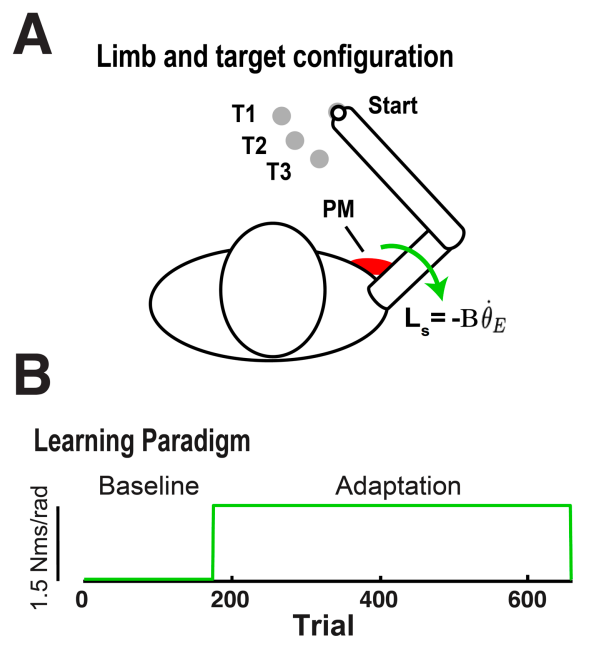

1176

1177

1178

1179

1180

1181

1182

1183

1184

1185

1186

1187

1188

1189

1190

1191

1192

1193

1194

1195

1196

1197

1198

Figure 1. Behavioural task and adaptation paradigm. $A$. Participants reached to two training targets $(\mathrm{T} 2, \mathrm{~T} 3)$ and a separate test target (T1). Green arrow represents novel shoulder loads $\left(L_{s}\right)$ applied in the adaptation block. $\boldsymbol{B}$. Time course of experiment. $\boldsymbol{C}$. Hand paths for exemplar good and partial adapters. $\boldsymbol{D}$. Average hand deviations at targets T1-T3 during baseline trials and last 25 adaptation trials. $E$. Average hand deviations from initial adaptation trials. ${ }^{*} p<$ 0.05 .
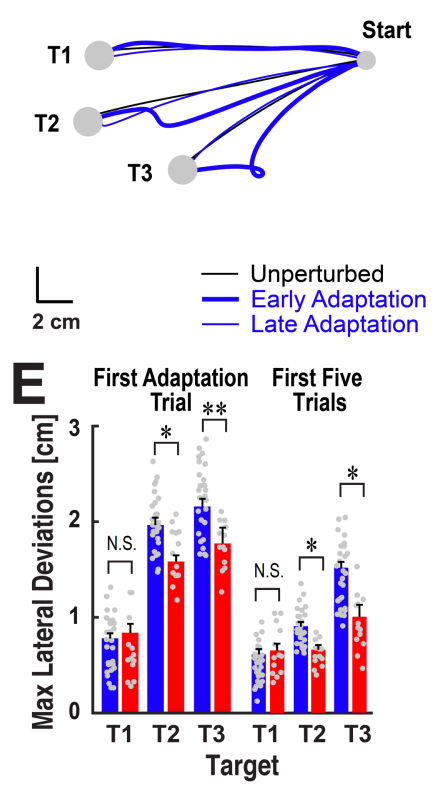
A

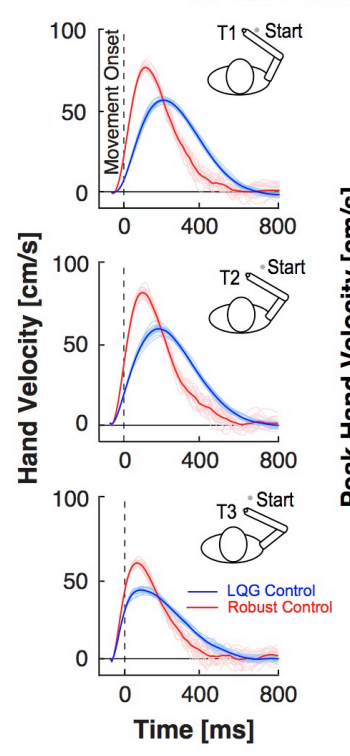

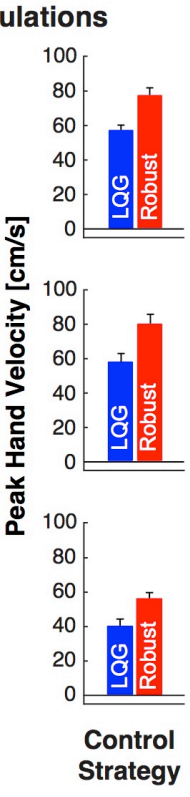

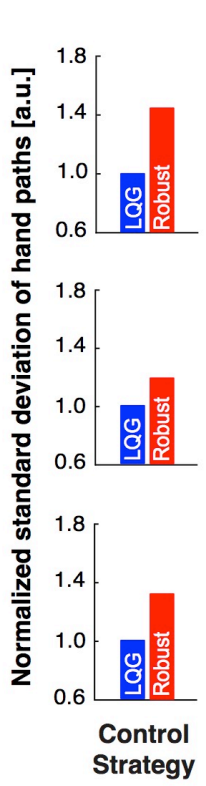

B
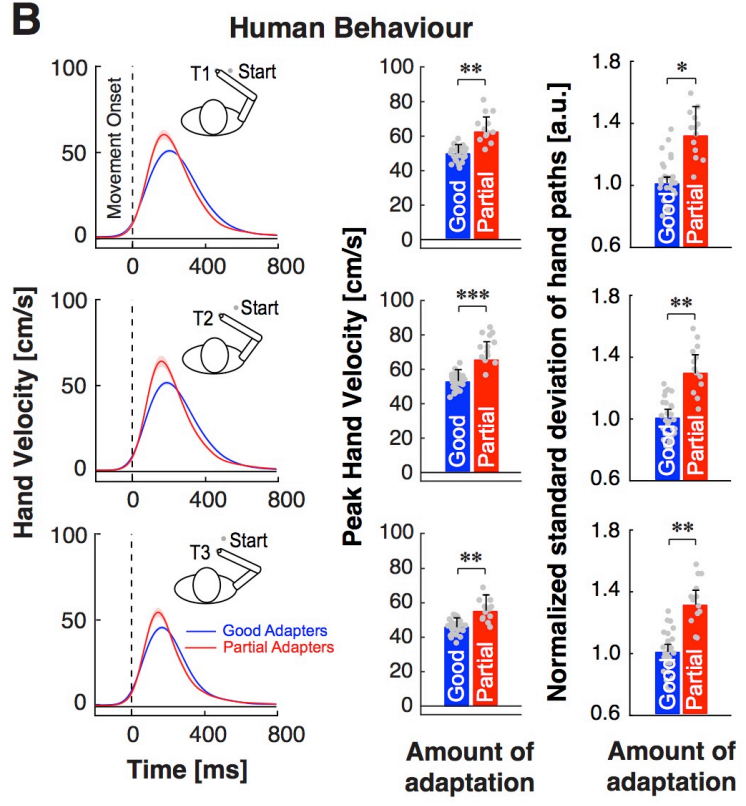

Figure 2. Baseline hand motion patterns. $\boldsymbol{A}$. Left: Simulated hand velocity profiles during baseline movements. Thin lines are individual trials; thick lines are average profiles. Right: Peak hand velocities and hand position variability. Data are normalized to variability of the stochastic optimal feedback controller (LQG). $B$. Left: Human hand velocity profiles (mean \pm SEM). Right: Peak hand velocities and position variability (mean \pm SEM). Data are normalized to the average variability displayed by good adapters. Dots represent individual participant averages. ${ }^{*} p<0.05,{ }^{* *} p<0.01,{ }^{* \star *} p<0.001$. 
A

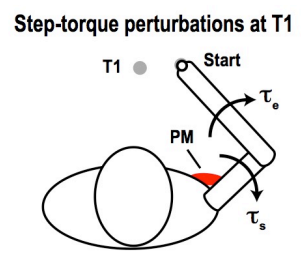

B

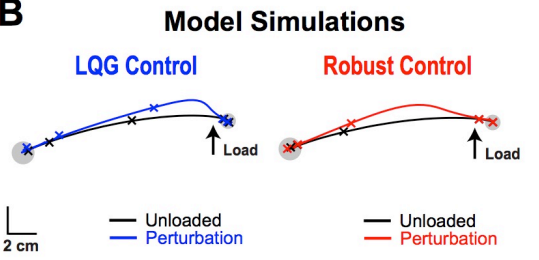

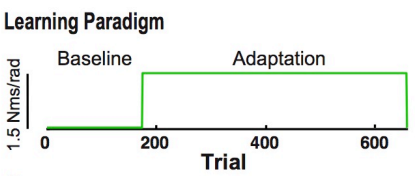
$\underset{N}{E} \sum_{\substack{\text { Interleaved perturbations at T1 } \\ \text { Int }}}^{||||||||||||||||||||||||||||||||||||||||||||||||}$

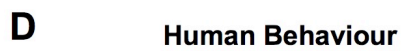

Exemplar Good Adapter Exemplar Partial Adapter
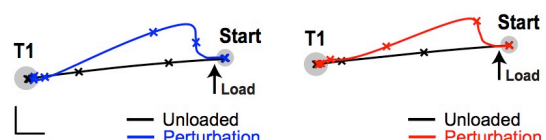

- Unloaded

C Shoulder Control Responses Peak Response
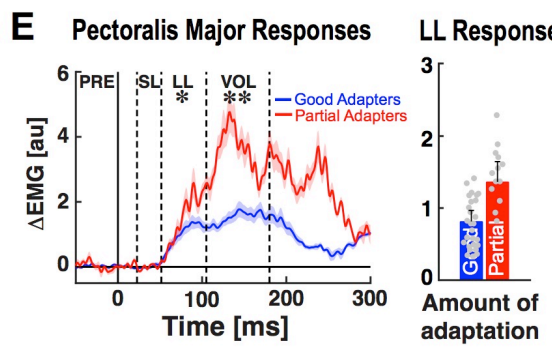

1224

1225

1226

1227

1228

1229

1230

1231

1232

1233

1234

1235

1236

1237

1238

1239

1240

1241

1242

1243

1244

1245

1246
Figure 3. Baseline feedback corrections. $\boldsymbol{A}$. Left: Schematic of behavioural task and applied step-torque perturbations. Right: Time course of experiment. Black lines show interleaved step-torque perturbations applied while reaching the test target (T1). B. Simulated hand paths during step-torque perturbation trials. $\boldsymbol{C}$. Shoulder control responses. Data are aligned to perturbation onset (Load ON). Bar graph shows the average peak in initial control response. $\boldsymbol{D}$. Hand motion patterns obtained from exemplar good and partial adapters during step-torque perturbation trials. Tick marks show the position of the hand every $200 \mathrm{~ms}$. $\boldsymbol{E}$. Pectoralis major stretch responses. Dashed vertical lines separate time phases of the muscle stretch response. Dots represent long-latency responses of individual participants. ${ }^{*} p<0.05,{ }^{* *} p<0.01$. 

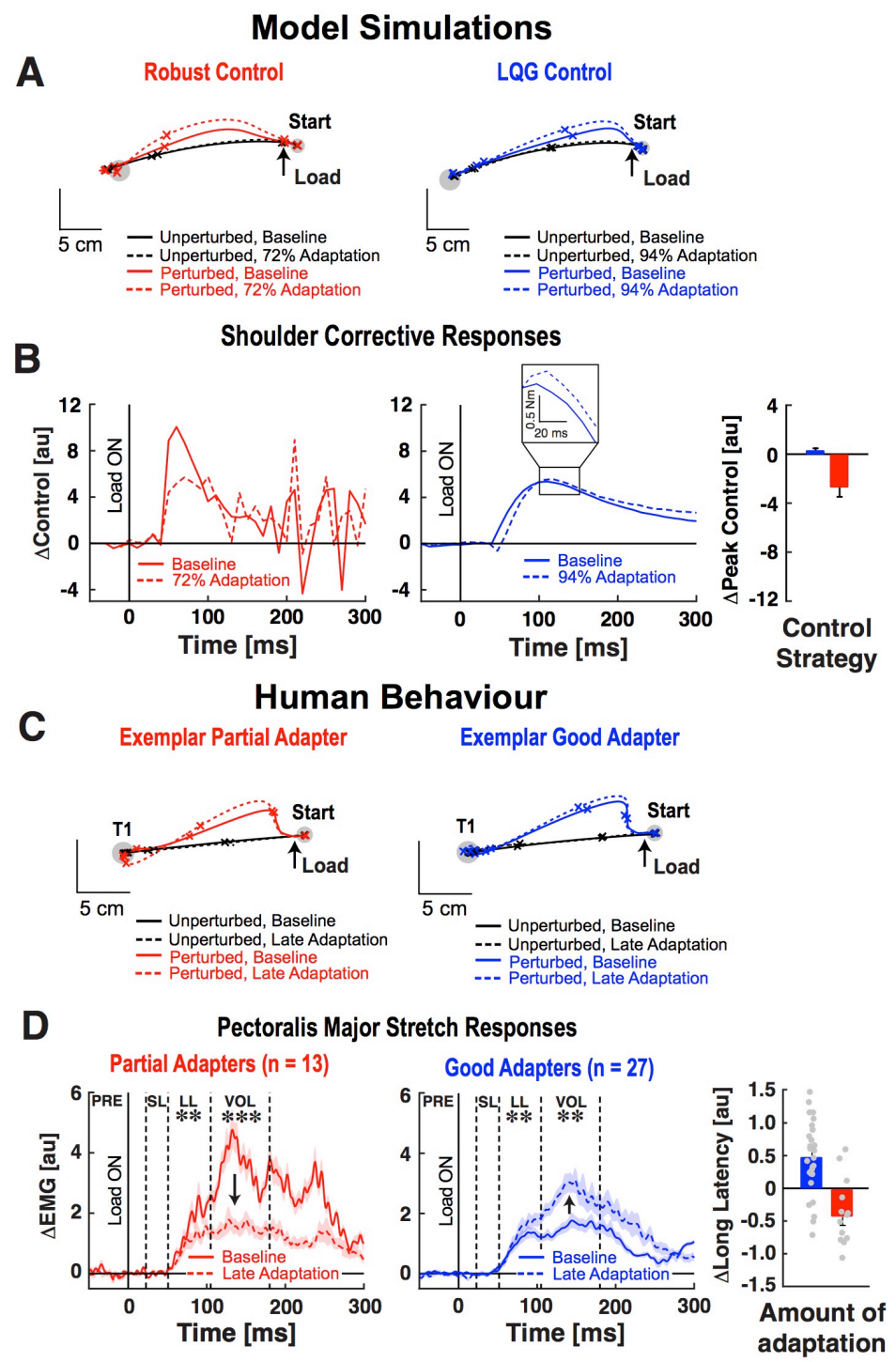

Figure 4. Comparison of baseline and adapted feedback responses. $A$. responses. Inset shows the LQG-control response. Bar graph shows average change in peak control response. $\boldsymbol{C}$. Hand motion obtained from exemplar good and partial adapters during step-torque perturbation trials. Tick marks show hand positions every $200 \mathrm{~ms}$. D. Pectoralis major stretch responses. Dashed vertical lines separate time phases of the muscle stretch response. Dots represent the change in long-latency response of individual participants. ${ }^{* *} p<0.01,{ }^{* * *} p<$ 
A Behavioural Task and Learning Paradigm
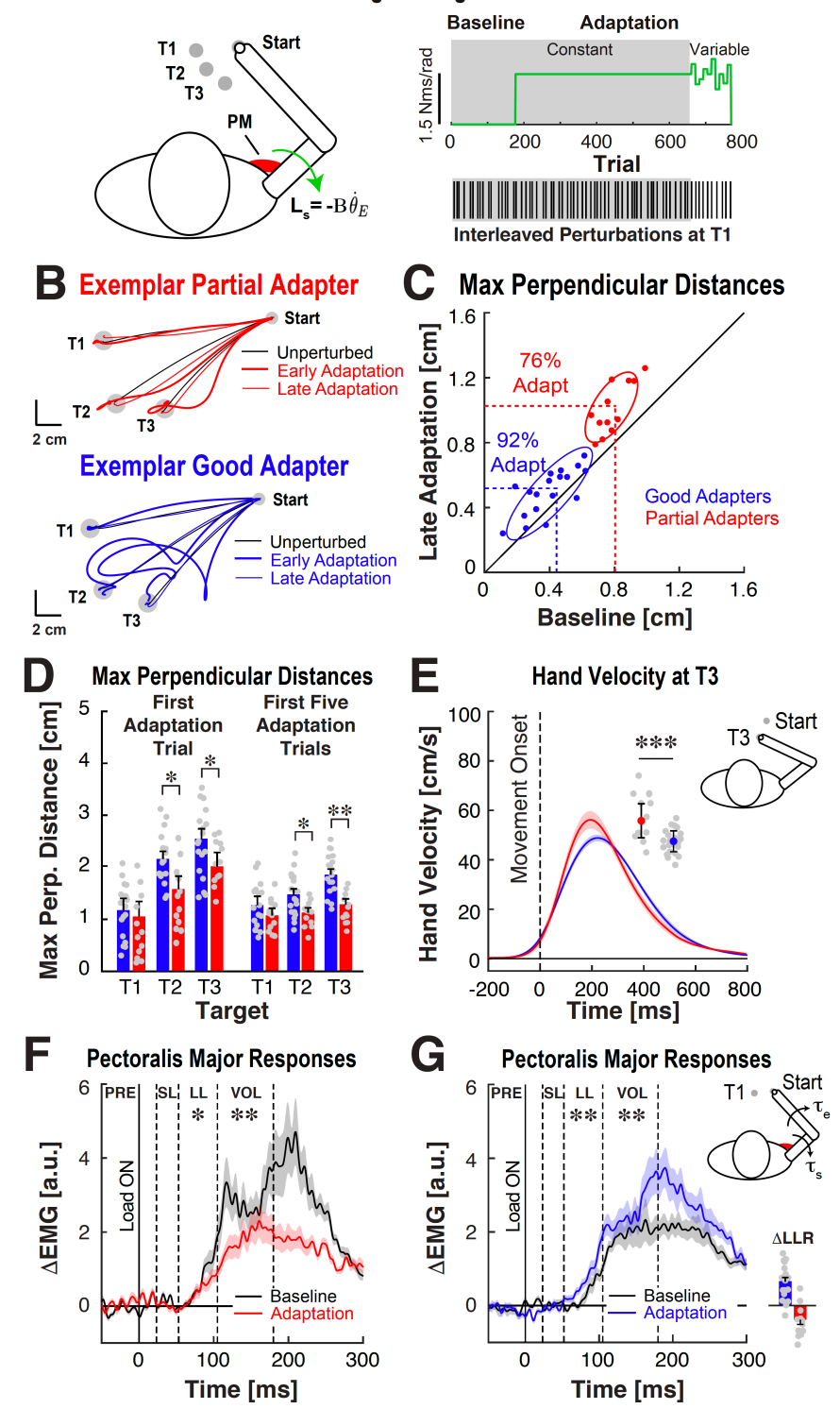

Figure 5. Behaviour and feedback corrections in Experiment 2. $A$. Behavioural task and time course of experiment. Participants reached to two training targets (T2, T3) and a separate test target (T1). Green arrow represents novel shoulder loads $\left(L_{s}\right)$ applied in the adaptation block. $\boldsymbol{B}$. Hand paths for exemplar partial and good adapters. $\boldsymbol{C}$. Average hand deviations at targets T1-T3 during baseline trials and last 25 adaptation trials at each target. $\boldsymbol{D}$. Average hand deviations from initial adaptation trials. $E$. Average velocity profiles observed for partial and good adapters. Error bars represent the SEM. Blue and red dots represent average peak velocity for groups of good and partial adapters $( \pm 1$ SEM). Grey dots represent individual subject averages. $\boldsymbol{F}$. Average pectoralis major stretch responses displayed by partial adapters. Dashed vertical lines separate time phases of the muscle stretch response. G. Average pectoralis major stretch responses displayed by good adapters. Data plotted in the same format as $\boldsymbol{F}$. Bar graphs represent average change in LLR between baseline and late adaptation. Dots represent individual participant data. ${ }^{*} p<0.05,{ }^{* *} p<0.01$. 


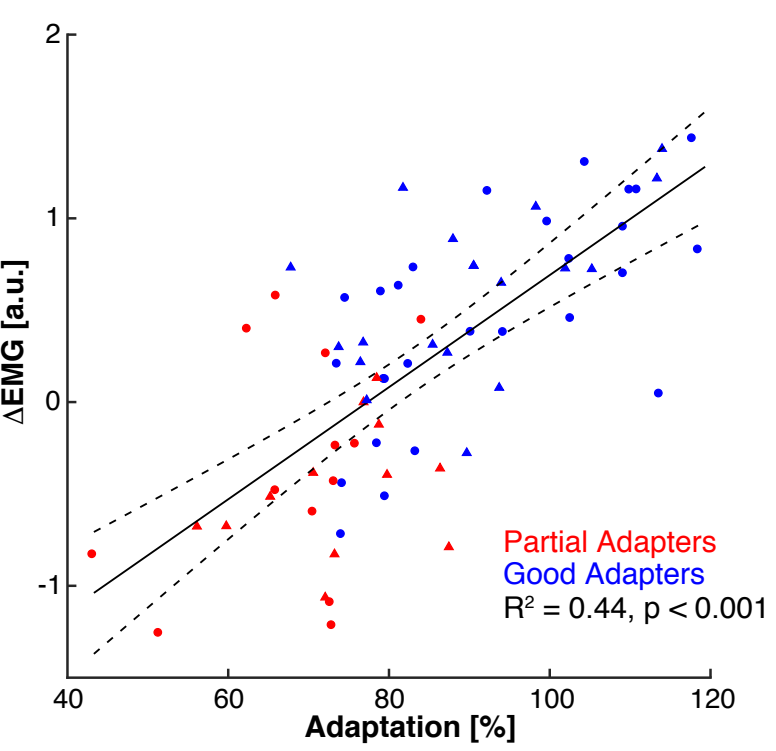

Figure 6. Relationship between adaptation (\%) and modulation of pectoralis major stretch responses between the baseline and late adaptation phases of the experiment $(\triangle E M G)$. Circles represent participants from Experiment 1, triangles represent participants from Experiment 2. 

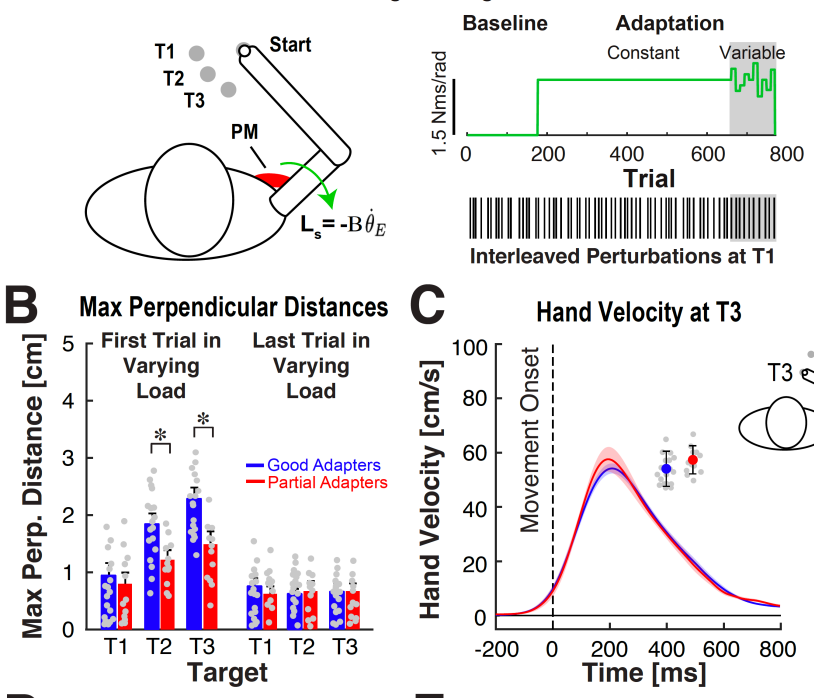

C Hand Velocity at T3
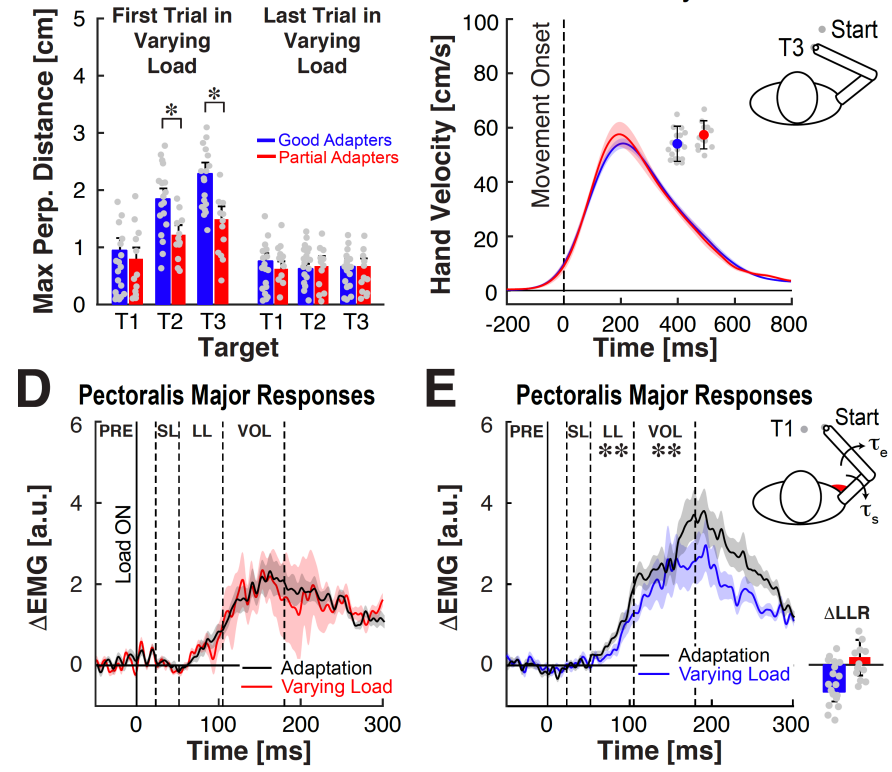

Figure 7. Behaviour and feedback corrections when exposed to frequent and abrupt changes in interaction loads. $\boldsymbol{A}$. Behavioural task and time course of experiment. $\boldsymbol{B}$. Average hand path deviations displayed by good and partial adapters when exposed to interaction dynamics that changed abruptly over short blocks of trials. $\boldsymbol{C}$. Average hand velocity profiles observed for partial $(n=12)$ and good adapters $(n=18)$. Error bars represent the SEM. Blue and red dots represent average peak velocity for groups of good and partial adapters. Grey dots represent individual subject averages. $\boldsymbol{D}$. Average pectoralis major stretch responses displayed by partial adapters when exposed to constant and varying interaction dynamics. Dashed vertical lines separate time phases of the muscle stretch response. E. Average pectoralis major stretch responses displayed by good adapters. Data plotted in same format as $\boldsymbol{D}$. Bar graphs represent average change in LLR. Dots represent individual participant data. ${ }^{*} p<0.05,{ }^{* *} p<0.01$, ${ }^{* * *} p<0.001$. 


\begin{tabular}{l|l|l} 
Parameter & Value [unit] & Justification \\
\hline Inertia (arm; forearm and hand) & $0.14,0.21\left[\mathrm{~kg} \mathrm{~m}^{2}\right]$ & Measured \\
Viscous friction & $0.14\left[\mathrm{Nm} \mathrm{s}^{-1}\right]$ & Reference $^{39}$ \\
Muscle time constant & $60[\mathrm{~ms}]$ & Reference $^{107}$ \\
Motor noise covariance $\left(\Sigma_{\xi}\right)$ & $0.025 \times B B^{T}$ & Qualitative match \\
Sensory noise covariance $\left(\Sigma_{\zeta}\right)$ & $\sigma^{2} I_{n \times n}$ & Reference \\
Feedback delay & $50[\mathrm{~ms}]$ & Latency of multi-joint FB
\end{tabular}

1342 Table 1: Numerical values of parameters and their justification. Remark: the 1343 value of $\sigma^{2}$ is equal to the elements of the noise covariance matrix of the motor 1344 noise. Thus the feedback noise has the same variance as the non-zero components of the motor noise, and the sensory covariance matrix has full rank in agreement with the assumptions of the Kalman filter ${ }^{110}$. 